Review

\title{
Symmetry-Related Electromagnetic Properties of Resonator-Loaded Transmission Lines and Applications
}

\author{
Jordi Naqui, Lijuan Su *, Javier Mata and Ferran Martín \\ GEMMA/CIMITEC, Departament d'Enginyeria Electrònica, Universitat Autònoma de Barcelona, \\ 08193 BELLATERRA, Barcelona, Spain; E-Mails: Jordi.Naqui@uab.cat (J.N.); \\ FranciscoJavier.Mata@uab.cat (J.M.); Ferran.Martin@uab.cat (F.M.) \\ * Author to whom correspondence should be addressed; E-Mail: Lijuan.Su@uab.cat; \\ Tel.: +34-93-581-35-22; Fax: +34-93-581-26-00.
}

Academic Editor: Takayoshi Kobayashi

Received: 12 February 2015 / Accepted: 7 May 2015 / Published: 18 May 2015

\begin{abstract}
This paper reviews the recent progress in the analysis and applications of the symmetry-related electromagnetic properties of transmission lines loaded with symmetric configurations of resonant elements. It will be shown that the transmission characteristics of these reactively loaded lines can be controlled by the relative orientation between the line and the resonant elements. Two main types of loaded lines are considered: (i) resonance-based structures; and (ii) frequency-splitting structures. In resonance-based transmission lines, a line is loaded with a single resonant (and symmetric) element. For a perfectly symmetric structure, the line is transparent if the line and resonator exhibit symmetry planes of different electromagnetic nature (electric or magnetic wall), whereas the line exhibits a notch (resonance) in the transmission coefficient if the symmetry planes behave as either electric or magnetic walls (symmetric configuration), or if symmetry is broken. In frequency-splitting lines, paired resonators are typically loaded to the transmission line; the structure exhibits a single notch for the symmetric configuration, whereas generally two split notches appear when symmetry is disrupted. Applications of these structures include microwave sensors (e.g., contactless sensors of spatial variables), selective mode suppressors (of application in common-mode suppressed differential lines, for instance) and spectral signature barcodes, among others.
\end{abstract}

Keywords: symmetry properties; transmission lines; microwave sensors; differential sensors; balanced lines; electrically small resonators; metamaterials; common-mode rejection 


\section{Introduction}

Transmission lines loaded with electrically small resonant elements, such as stepped impedance resonators (SIRs) [1], split ring resonators (SRRs) [2-5], spiral resonators [6], and other resonators related to the previous ones, including the complementary (or slotted) counterparts [7-11], have been applied to the implementation of planar microwave filters [1,8,12,13], spurious suppression [14], metamaterial-inspired devices [15,16], microwave sensors [17-21], radiofrequency barcodes [22,23], etc. In most of these applications, the resonator is electrically and/or magnetically coupled to the line, providing a notch at the fundamental resonance frequency of the resonant element. However, the fact that the resonant elements are etched close to the line does not guarantee that (effective or net) line-to-resonator coupling arises. Indeed, the relative orientation between the line and the resonant element is a key factor to determine whether coupling is present or not, and the nature (electric, magnetic or mixed) of such coupling. For instance, it was demonstrated in [24] that in coplanar waveguide (CPW) transmission lines loaded with pairs of SRRs symmetrically etched in the slot regions, mixed coupling (electric and magnetic) arises unless the SRRs are etched with their symmetry planes orthogonally oriented to the line axis (in that case, the coupling between the line and the SRRs is only magnetic). Similarly, in microstrip lines loaded with complementary split ring resonators (CSRRs), the line-to-resonator coupling is electric for orthogonal orientation, and mixed coupling appears for any other particle orientation (see Figure 1). Both SRRs and CSRRs are bianisotropic particles [25] that can be excited by either a time-varying electric or magnetic field (conveniently oriented), and for that reason particle orientation with regard to the line is fundamental to control the nature of coupling.

(a)

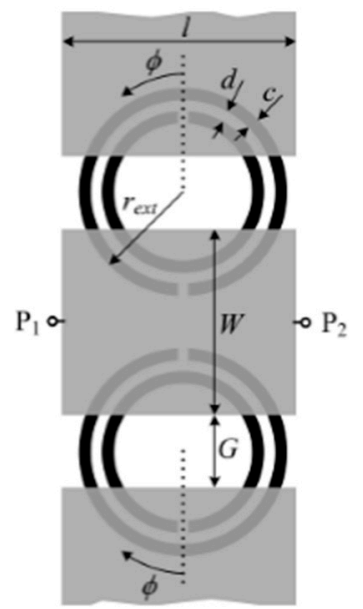

(b)

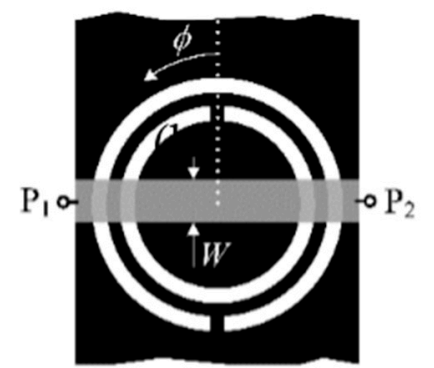

Figure 1. (a) CPW (Coplanar waveguide) transmission line loaded with a pair of SRRs (split ring resonators) with the symmetry plane orthogonally oriented to the line axis; (b) Microstrip transmission line loaded with a CSRR (complementary split ring resonator) with the symmetry plane orthogonally oriented to the line axis. If the particles are rotated $(\phi \neq 0)$, mixed coupling arises.

However, resonator orientation and position are not only important to control the coupling mechanism, but also to control the presence and level of coupling in certain lines loaded with single symmetric resonant particles [19], or to control the number of notches in the transmission coefficient in transmission lines loaded with pairs of resonant elements [26]. In this work, it will be shown that particle 
orientation and symmetry are fundamental to control the transmission properties of resonator loaded lines. Moreover, an exhaustive analysis, on the basis of lumped element equivalent circuit models of the considered structures, is carried out. Moreover, some applications derived from the symmetry related electromagnetic properties of these lines mainly based on selective mode suppression [27-29] or symmetry disruption $[19,30]$ will be pointed out, including common-mode suppressed balanced lines, microwave sensors for spatial (angular) measurement, and microwave differential permittivity sensors (actually comparators).

The work is organized as follows. In Section 2, the symmetry-related electromagnetic properties that lead us to the control of the transmission characteristics in transmission lines loaded with symmetric configurations of resonant elements are explained in detail. In Section 3, the circuit models of certain representative structures are presented. The considered structures are differential microstrip lines loaded with CSRRs, CPW transmission lines loaded with electric LC (ELC) resonators, and CPWs loaded with pairs of SRRs. Section 4 is devoted to the applications. Finally, the main concluding remarks are summarized in Section 5.

\section{Theory and Principle}

Let us now focus on the transmission characteristics of resonator loaded lines and the role of symmetry. The two types of structures considered are: (i) lines loaded with a single resonator, where a single notch (resonance) may appear or not depending on particle orientation and position; and (ii) lines loaded with a pair of resonant elements, where disruption of symmetry leads to frequency splitting.

\subsection{Lines Loaded with a Single Symmetric Resonator (Resonance-Based Structures)}

In general, if a planar resonant element is etched in close proximity to a transmission line, coupling between the line and the resonator arises, and the line prevents the transmission of signals at the fundamental resonance frequency and at higher-order resonance frequencies. Depending on the line and resonator topology, orientation, and position, the line-to-resonator coupling may be electric, magnetic, magneto-electric (or mixed), or it may be cancelled (in this case providing total transmission at the resonance frequency). From now on, we will focus the attention only on frequencies in the vicinity of the fundamental resonance frequency of the resonant element. In lines loaded with a single and symmetric resonant element, and assuming that the resonator is under the influence of the field generated by the line, a necessary condition to prevent line-to-resonator coupling is a perfect alignment between the line axis and the symmetry plane of the resonator. Under these circumstances, if the symmetry planes of the line and resonator are of different electromagnetic nature (one an electric wall and the other one a magnetic wall), then net coupling is cancelled, the resonator is not excited, and the line is transparent $[19,27]$. Conversely, if the symmetry planes are of the same electromagnetic nature (either an electric wall or a magnetic wall) the resonator is driven and the transmission coefficient exhibits a resonance (notch). Note that certain lines support more than one fundamental mode of propagation (e.g., differential microstrip lines may propagate even- and odd-modes), and hence the electromagnetic nature of the symmetry plane depends on the considered propagating mode. This means that in such lines, selective mode suppression is possible [27], as will be discussed later. If the symmetry planes of the line and resonator are misaligned, then, in general, line-to-resonator coupling arises. 
Note that in the context of this paper an electric/magnetic wall is a virtual plane with an anti-symmetric/symmetric charge distribution at both sides. Besides, even- and odd-modes are symmetric and anti-symmetric modes, respectively, that propagate in symmetric structures, such as in a symmetric pair of coupled lines. In summary, the line-to-resonator coupling may be controlled or modulated, which in turn modulates the associated resonance. According to this feature, a possible designation for such structures may be coupling-modulated resonance-based structures or simply resonance-based structures.

To clarify the previous assertions related to the coupling (resonance) prevention, consider as an illustrative example a CPW transmission line (see Figure 2). The fundamental CPW mode is an even mode and hence the transmission line exhibits a magnetic wall at its symmetry plane. If we align with such a line an SRR (it exhibits an electric wall at its symmetry plane at the fundamental resonance), the resonator will not be excited (Figure 2a). In terms of fields, for this configuration there is a perfect cancellation of magnetic fields in the inner region of the SRR, and the resonator is not excited because there is no net magnetic field penetrating the SRR [19]. However, for the slot (odd) mode of the CPW, the resonator will be excited, since the symmetry plane of the line is an electric wall for that mode (Figure 2b), and a net magnetic field in the SRR, able to induce electric currents in the particle, arises. Thus, selective mode suppression in the vicinity of the SRR fundamental resonance is possible [28]. It will be shown later (Section 4) that selective mode suppression in balanced microstrip lines is possible by using CSRRs, and particularly useful for common-mode suppressed balanced lines.

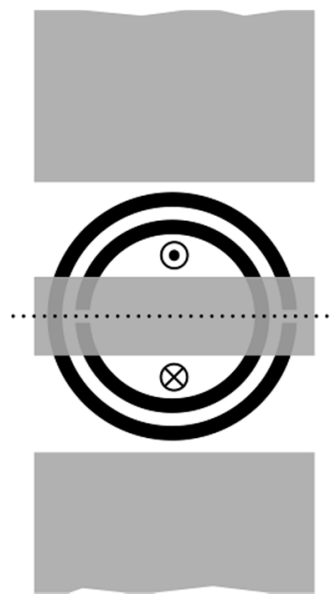

(a)

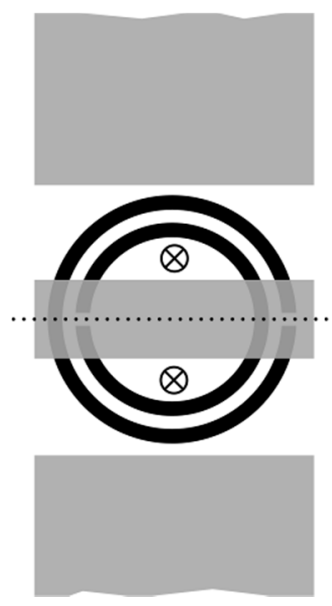

(b)

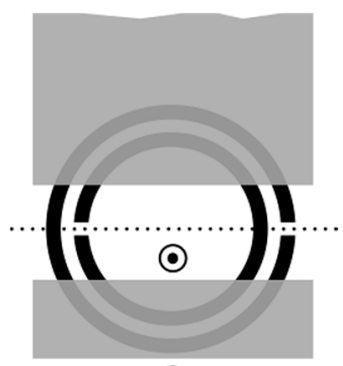

$\otimes$

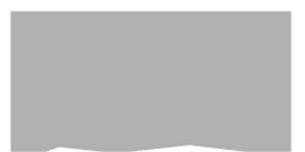

(c)

Figure 2. CPW transmission line loaded with a single SRR. (a) Line and resonator aligned and excitation with the fundamental (even) mode; (b) line and resonator aligned and excitation with the slot (odd) mode; (c) line and resonator misaligned and excitation with the fundamental (even) mode. The cross and dot point to the direction of the magnetic field axial to the SRRs.

Finally, if the resonator is not aligned with the line, i.e., rotated or laterally displaced (Figure 2c), perfect cancellation of fields in the particle is not possible and a notch in the transmission coefficient emerges (exceptionally, the structure may be still transparent when only a small amount of field lines illuminates the resonator). Due to symmetry disruption, the resonator is effectively coupled to the line, the coupling level (and hence the notch magnitude) being intimately related to the level of asymmetry. 
From the previous words, it follows that transmission lines loaded with movable symmetric resonators can be used for sensing linear and angular displacements and velocities. Obviously, the line and resonator geometry need to be optimized in order to achieve optimum sensor performance (linearity, sensitivity, dynamic range, etc.). This depends on the specific type of measurement and is left for Section 4, where various examples are reported. Since the symmetry is not affected by changes in environmental conditions, these sensors based on symmetry properties are robust against variable ambient conditions (temperature, moisture, etc.) [30-33]. It should be highlighted that these sensors are especially suited to detecting the lack of alignment between the resonator and the line, and are therefore useful as alignment sensors. Symmetry disruption can also be achieved by means of different mechanisms (not only by linear or angular displacements), for instance, by asymmetric loading of additional inclusions. Thus, these structures may be of interest as comparators or sensors for dielectric measurements [33], where small samples of dielectric materials are loaded to the structure. Figure 3 illustrates the different possibilities concerning discrete loading, where it can be appreciated that symmetry rupture generates a notch in the transmission coefficient. Lastly, it is also worth pointing out that frequency-domain barcodes implemented by multi-resonating structures [22] and using the resonance-based approach have also been reported [33].
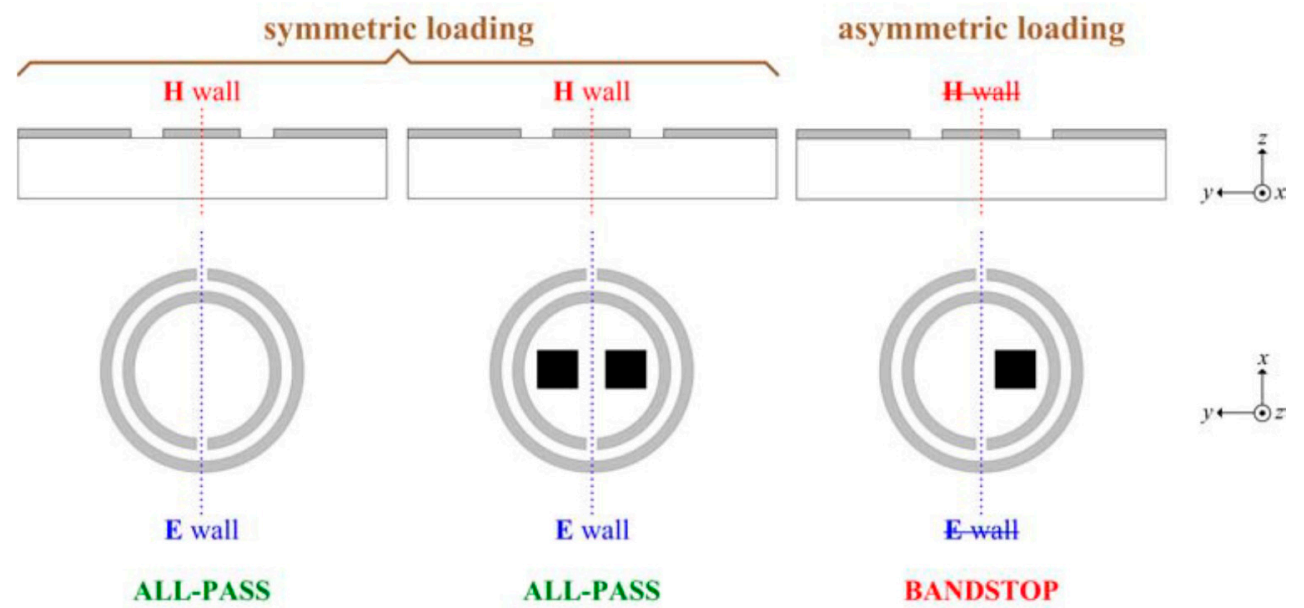

Figure 3. Illustration of symmetry rupture by square-shaped inclusions in the approach based on a single resonator, where a CPW is loaded with a symmetrically oriented SRR.

\subsection{Lines Loaded with a Pair of Resonators (Frequency-Splitting Structures)}

In lines loaded with a pair of resonant elements (e.g., Figure 1a), the symmetric configuration leads to a single transmission zero, since the fundamental resonance frequencies of the two resonators degenerate to the same one. However, if the whole structure is asymmetric, in general there are two transmission zeros because two different resonances arise. This is here referred to as frequency splitting, meaning that the electromagnetic structure exhibits two split or separated resonance frequencies. It should be also stressed that the two resonance frequencies depend on the resonance frequencies of the two resonators, as well as on the coupling between the resonators, if present. It should be noted that in transmission lines loaded with pairs of resonators (transversally arranged), line-to-resonator coupling for both resonators cannot be canceled simultaneously. Therefore, resonance condition always occurs (as long as the resonator is in close proximity to the transmission line, which is obvious). 


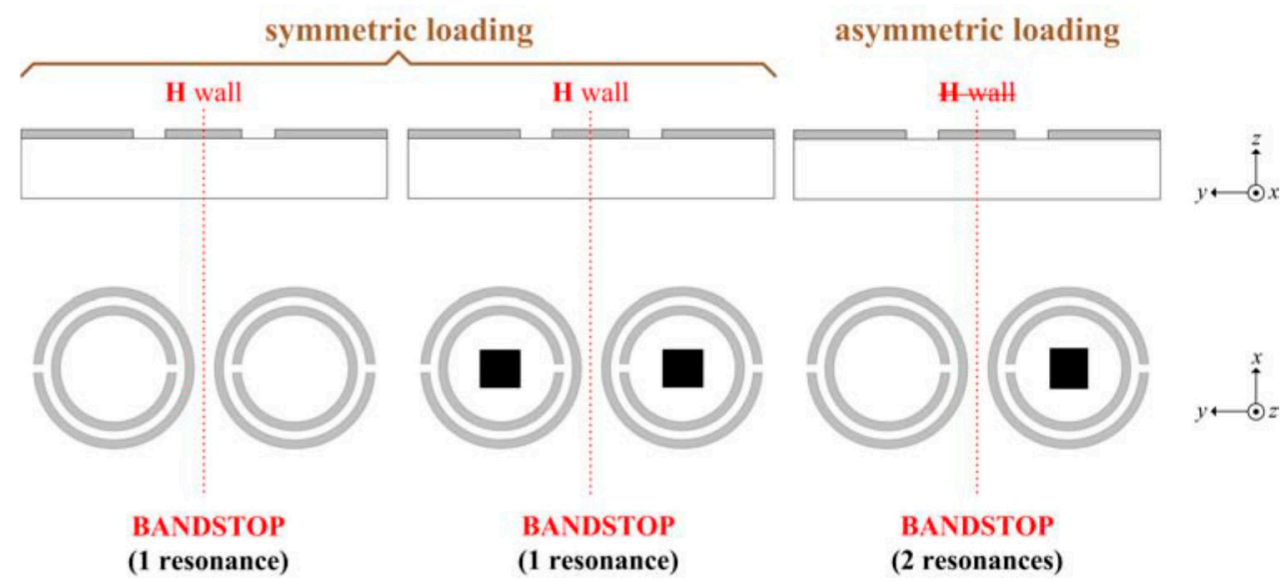

Figure 4. Illustration of symmetry rupture by square-shaped inclusions in the approach based on pairs of resonators, where a CPW is symmetrically loaded with a pair of SRRs. Note that the symmetry planes of the SRRs are not required to be parallel to the longitudinal $x$-direction, preventing mixed coupling.

Consequently, in this approach based on pairs of resonators, a high controllability in regard to the number and frequency of resonances as a function of symmetry may be achieved (the bandwidth and depth of the notches, however, may also depend on symmetry). Figure 4 illustrates the above-mentioned assertions where symmetry is disrupted by inclusions. As can be seen, an interesting aspect of this approach is that there is no restriction in the orientation of the individual resonators in regards to the line. This is important for cancel mixed-coupling effects in certain topologies [24]. For example, if symmetric SRRs are considered, their symmetry planes are not needed to be oriented longitudinally and the SRRs are coupled only magnetically to the line.

Like in the strategy based on single resonators, symmetry rupture by adding inclusions to the structure using paired resonators may be useful for the implementation of differential sensors and comparators [34]. Potential applications are dielectric characterization, quality control (e.g., defect detection in samples by comparison to a reference), biosensors, etc. It is also possible to use this sensing principle based on frequency splitting for the implementation of spatial sensors [35].

\section{Circuit Models}

In this section, the circuit models of some representative structures of resonance-based and frequency splitting structures are provided and validated. As is well known, the accuracy of the models is subjected to the electrical size of the considered resonant elements.

\subsection{Differential Microstrip Lines Loaded with CSRRs}

Differential microstrip lines exhibit two fundamental modes, the even and the odd mode. Hence, the symmetry plane is an electrical wall for the odd mode and a magnetic wall for the even mode. This means that if a CSRR is symmetrically etched in the ground plane (i.e., with its symmetry plane aligned to that of the line), as Figure 5a illustrates, the even mode is expected to be suppressed in the vicinity of the fundamental resonance, whereas the line is expected to be transparent for the odd mode. The reason is that the CSRR exhibits a magnetic wall at the fundamental resonance frequency. The circuit model of 
this structure is depicted in Figure 5b [36]. The element $L$ models the inductance of the lines, $C$ accounts for the electric coupling between the lines and the resonator (and the line capacitance as well), and the CSRR is modeled by the parallel resonant tank, $L_{c}-C_{c}$. Finally, $C_{m}$ and $L_{m}$ model the mutual capacitance and inductance between the coupled lines, respectively.

The circuit model of Figure 5b explains that the differential signals are insensitive to the presence of the CSRRs, while these resonators prevent the transmission of the common mode at the fundamental resonance frequency of the CSRRs. The simplified equivalent circuit model under common-mode excitation is depicted in Figure 6a, whereas that for the odd mode is depicted in Figure 6b. For the odd mode, the resonator is short circuited to the ground, and the resulting model is the one of a conventional transmission line. For the even mode, we obtain the same circuit as the one of a CSRR-loaded line [37], but with modified parameters. Thus, a similar stop band behavior for the common mode is obtained.

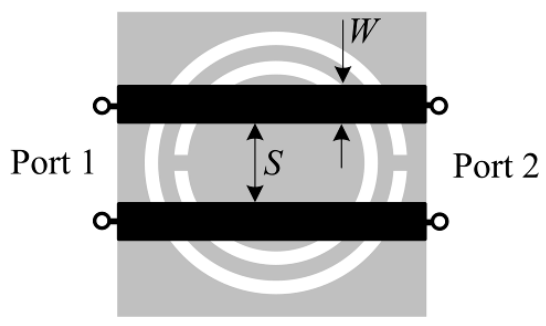

(a)

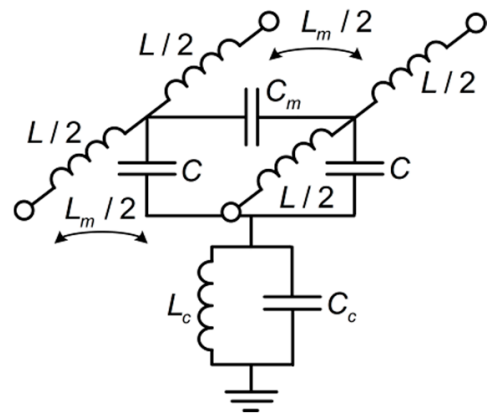

(b)

Figure 5. Topology (a) and circuit model (b) (elemental cell) of a differential microstrip line loaded with a CSRR.

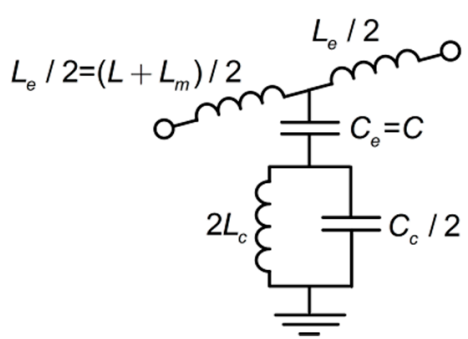

(a)

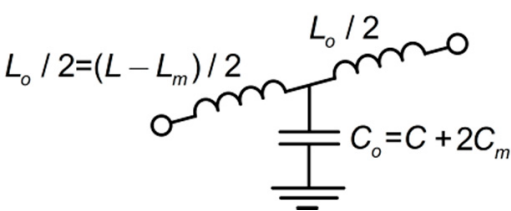

(b)

Figure 6. Circuit models for the even mode (a) and odd mode (b).

To validate the model, we have considered a CSRR unit cell (see the inset in Figure 7) with narrow and closely spaced apart slot rings $(c=0.2 \mathrm{~mm}$ and $d=0.2 \mathrm{~mm})$. These values are close to the limit of the available technology and guarantee that the particle is electrically small. With these values, the model of the CSRR reported in [37], and the per-unit length capacitance of the coupled lines (common mode), we have estimated the side of the CSRR in order to obtain a transmission zero frequency at $f_{z}=1.4 \mathrm{GHz}$ (obviously, optimization has been required since the model reported in [37] is valid under conditions not exactly fulfilled in the structure under study). The simulation of the common-mode insertion loss is depicted in Figure 7. The circuit simulation of the structure with the parameters extracted according to the procedure reported in [38] is also depicted in Figure 7, and good agreement with the electromagnetic simulation is noticeable. In order to enhance the rejection bandwidth, an order-three structure with tightly 
coupled CSRRs has been implemented [36]. The bandwidth is closely related to the level of inter-resonator coupling. Therefore, we have separated $0.15 \mathrm{~mm}$ the CSRR in order to broaden the bandwidth as much as possible. The simulation of the resulting structure is shown in Figure 8, where for comparison purposes we have also included the circuit simulation. The simulated circuit is that resulting by cascading the elemental cells, but with the addition of coupling capacitances between adjacent resonators. The coupling capacitance has been considered to be an adjustable parameter, and we have found that the capacitance that provides a better fit is $C_{R}=0.11 \mathrm{pF}$. The dimensions of the active (slot) region are $23 \mathrm{~mm} \times 7.6 \mathrm{~mm}$, that is $0.28 \lambda \times 0.09 \lambda$ (where $\lambda$ is the guided wavelength at the central frequency). The device is therefore very small, although bandwidth has not been optimized in this structure. It is clear that the differential signal is not altered by the presence of the CSRRs (see also Figure 8).

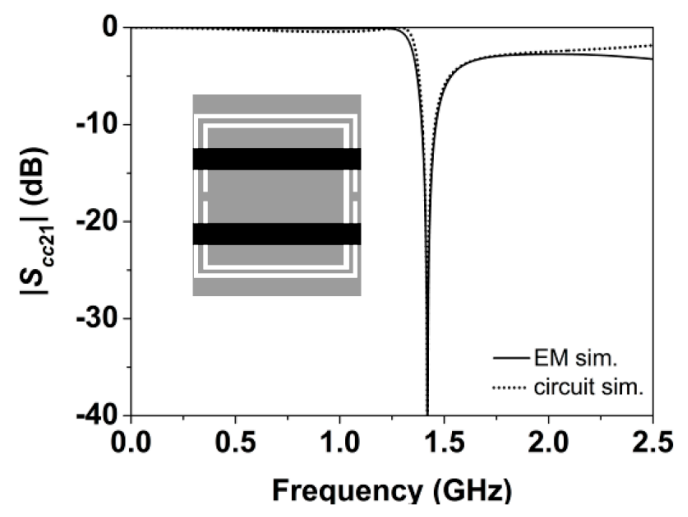

Figure 7. Simulated common-mode insertion loss $\left|S_{c c 21}\right|$ for the structure shown in the inset. CSRR dimensions are: $c=0.2 \mathrm{~mm}, d=0.2 \mathrm{~mm}, W=1 \mathrm{~mm}, S=2.5 \mathrm{~mm}$, and side length of $7.6 \mathrm{~mm}$. The considered substrate is Rogers RO3010 with thickness $h=1.27 \mathrm{~mm}$ and dielectric constant $\varepsilon_{r}=10.2$. Extracted parameters are $L_{e}=9.86 \mathrm{nH}, C=1.06 \mathrm{pF}$, $C_{c}=2.68 \mathrm{pF}$ and $L_{c}=3.36 \mathrm{nH}$. From [36]; reprinted with permission from IEEE 2011.

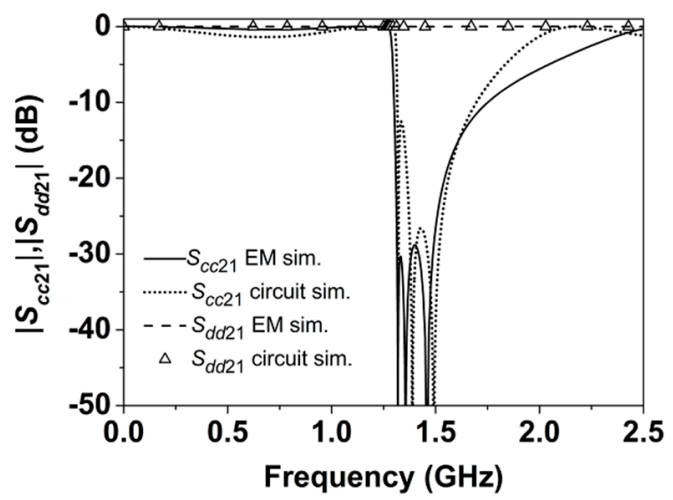

Figure 8. Simulated common-mode and differential-mode insertion loss $\left|S_{c c 21}\right|$ and $\left|S_{d d 21}\right|$ for the order-three structure that results by cascading three-unit cells like the one shown in Figure 7. Inter-resonator distance is $0.15 \mathrm{~mm}$. From [36]; reprinted with permission from IEEE 2011.

\subsection{CPWs Loaded with ELC Resonators}

The ELC resonator (Figure 9) is a bisymmetric resonant particle [39]. At the fundamental resonance frequency, the currents in the two arms flow in opposite directions (one is clockwise and the other one 
is counterclockwise, as Figure 9 illustrates). The particle can be excited by means of a time-varying uniform electric field applied to the direction orthogonal to the electric wall ( $y$-direction), but it cannot be excited by means of a uniform magnetic field polarized in the $z$-direction, since there is not a net magnetic dipole in that direction at the fundamental resonance. However, the particle can be excited by means of counter-magnetic fields applied to the two loops, as Figure 9 shows. From these assertions, it follows that if the particle is etched on the back side of a CPW transmission line (or on top of it), it can be excited depending on its relative orientation with regard to the line [40,41]. If the magnetic wall of the particle is aligned with the line axis (also a magnetic wall for the fundamental CPW mode), the ELC will be excited and a notch in the transmission coefficient is expected. Conversely, the CPW will be transparent if the ELC is oriented with the electric wall aligned with the line axis. Obviously for intermediate angles, the particle will be coupled to the line and the coupling strength can be controlled by the angle. These ELC-loaded lines are therefore useful as rotation sensors for alignment, displacement and velocity measurements, as will be shortly discussed.

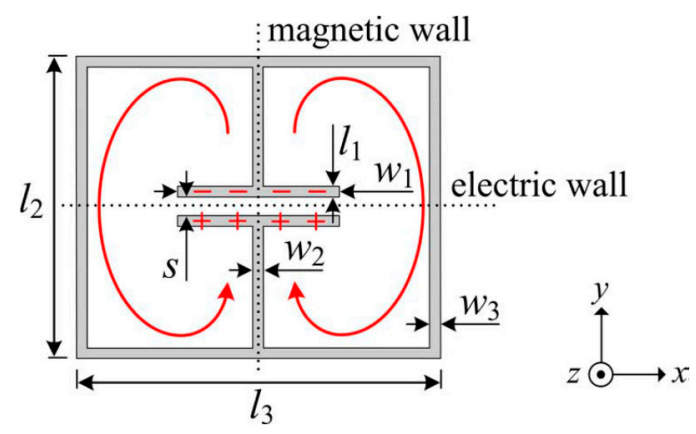

Figure 9. Bisymmetric ELC (electric-LC) resonator. The electric and magnetic walls at the fundamental resonance, as well as a sketch of the distribution of charges and currents, are indicated.

The proposed equivalent circuit model of an ELC-loaded CPW is depicted in Figure 10a [41]. This circuit is valid as long as the cell length is electrically small (losses are not considered). The ELC resonator is represented by the inductances $L_{e}$ and the capacitance $C_{e}$, modeling the inductive loops and the capacitive gap, respectively. The CPW is modeled by its inductance and capacitance, $L$ and $C$, respectively, and it is divided into two identical halves for convenience. Finally, each half is magnetically coupled to each loop through the mutual inductances $M_{\theta}$ and $M^{\theta}$, both being dependent on the angle $\theta$ (different dots are used to distinguish the magnetic coupling sign associated to each of the halves). Therefore, the frequency response of the circuit of Figure 10a directly depends on the angle $\theta$. When $\theta=0^{\circ}$, due to symmetry, $M_{\theta}=M^{\theta} \neq 0$, which means that the currents flowing on the CPW induce a pair of equal and anti-phase voltages in the loops; no net voltage is induced in the resonator due to an absolute cancellation. In other words, there is not a net magnetic coupling, the resonator cannot be magnetically driven, and the resulting model is that of a conventional transmission line. On the other hand, as $\theta$ increases, the magnetic coupling is complementarily distributed; $M^{\theta}$ increases at the expense of a decrease in $M_{\theta}$. Hence for $\theta>0^{\circ}$, a net induced voltage arises and the line is indeed capable of magnetically exciting the resonator. The larger the angle, the higher the induced voltage. Thus, at the upper limit, $\theta=90^{\circ}, M^{\theta}$ is maximum while $M_{\theta}$ vanishes completely; the magnetic coupling cancellation disappears and the resonator is expected to be tightly coupled to the line. 


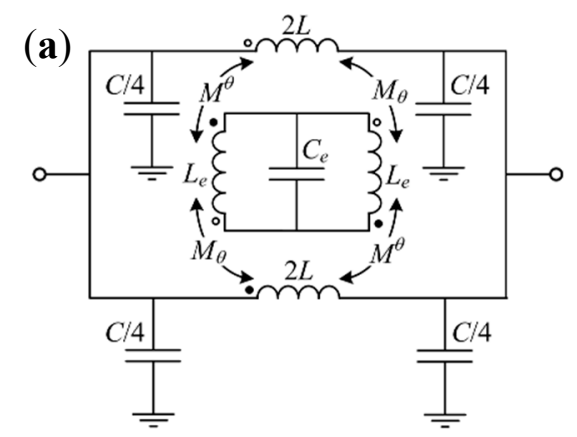

(b)

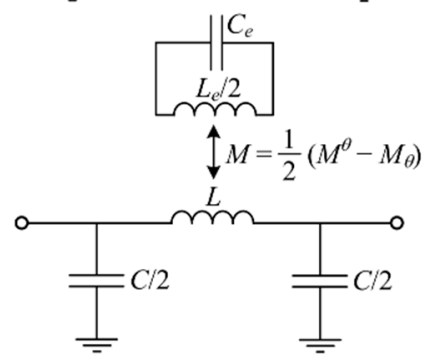

(c)

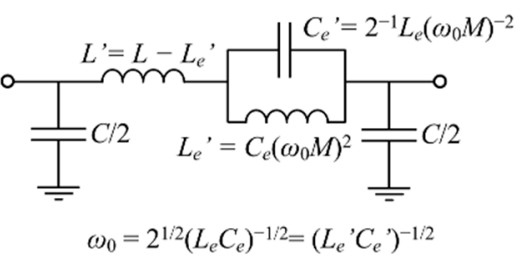

Figure 10. (a) Equivalent circuit model of a CPW loaded with an ELC resonator, including the different magnetic coupling mechanisms, accounted for through the mutual inductances $M_{\theta}$ and $M^{\theta}$; (b) simplified circuit model; (c) transformed simplified circuit model.

The circuit of Figure 10a is somewhat complex and has too many parameters for both parameter extraction and design purposes. An equivalent and simplified circuit model is the one depicted in Figure $10 \mathrm{~b}$, where an effective mutual inductance $M$ is defined. Such model is equivalent to that of an SRR magnetically coupled to a CPW transmission line, which can be transformed to the circuit model of Figure 10c $[42,43]$. From the latter model, it can be concluded that not only there is a transmission zero at $f_{0}=\omega_{0} / 2 \pi$ as long as $M$ is different from zero, but also the rejection bandwidth broadens with $M$ (the reason is that the susceptance slope of the parallel resonator decreases as the ratio $L_{e}{ }^{\prime} / C_{e}$ ' increases). Furthermore, the lumped element values of that circuit can be easily extracted from the method reported in [43].

To deal with different orientations, we will consider a circular-shaped structure as a case study (see Figure 11) [41]. The capacitance of the ELC is enhanced by using the semi-circular metallic patches, thus resulting in a relatively small electrical size. The results of the electromagnetic simulation for different resonator orientations are plotted in Figure 12. The extracted parameters for the circular structure (under different orientations) are listed in Table 1. It is remarkable that with the exception of the mutual inductance, all the circuit parameters are roughly invariant with the rotation angle. The reflection and transmission coefficients inferred from the circuit simulation with the extracted parameters are also depicted in Figure 12. Good agreement between the circuit and the electromagnetic simulations in the vicinity of the transmission zero frequency (i.e., the region of interest) results, thus validating the proposed circuits with arbitrary ELC orientation. 


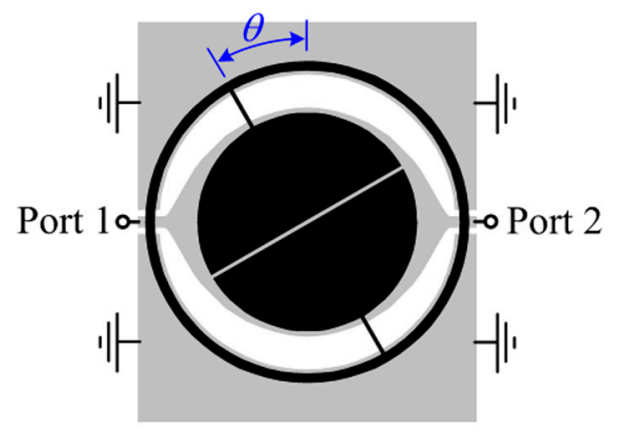

Figure 11. Layout of the circular-shaped ELC-loaded CPW structure (for a specific angle of $30^{\circ}$ ). The considered substrate is Rogers RO3010 with $h=1.27 \mathrm{~mm}$ and $\varepsilon r=11.2$. The dimensions are: for the line, $W$ and $G$ are tapered such that the characteristic impedance is $50 \Omega$; for the ELC resonator: mean radius $r_{0}=8.05 \mathrm{~mm}$, capacitor outer radius $r_{1}=11 \mathrm{~mm}$, $w_{2}=s=0.2 \mathrm{~mm}$, and $w_{3}=0.5 \mathrm{~mm}$.
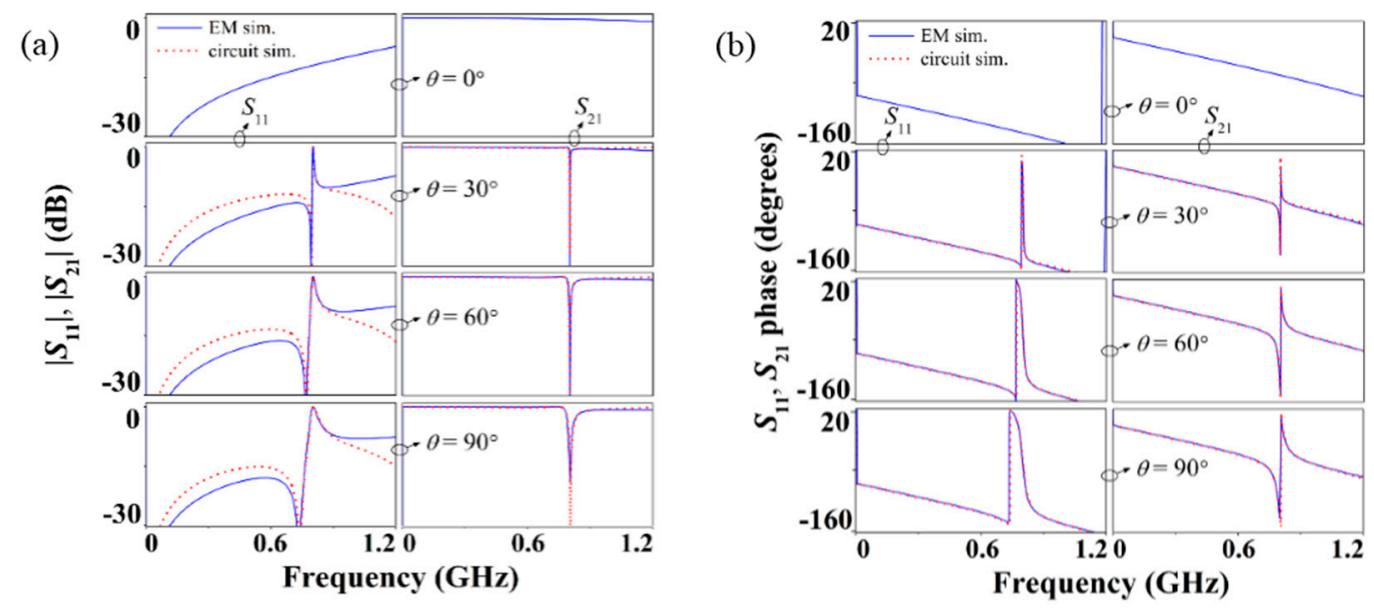

Figure 12. (a) Magnitude and (b) phase of the reflection and transmission coefficients given by the lossless electromagnetic simulation for the structure of Figure 11 and by the circuit simulation for the models of Figure 10. The circuit parameters are indicated in Table 1. From [41]; reprinted with permission from IEEE 2013.

Table 1. Extracted lumped element values of the circuit of Figure $10 \mathrm{~b}$ for the structure of Figure 11.

\begin{tabular}{cccccc}
\hline $\boldsymbol{\theta}$ (Degrees) & $\boldsymbol{C}(\mathbf{p F})$ & $\boldsymbol{L}(\mathbf{n H})$ & $\boldsymbol{C}_{\boldsymbol{e}}(\mathbf{p F})$ & $\boldsymbol{L}_{\boldsymbol{e}}(\mathbf{n H})$ & $\boldsymbol{M}(\mathbf{n H})$ \\
\hline 30 & 5.86 & 5.95 & 3.09 & 25.6 & 0.94 \\
60 & 5.73 & 6.22 & 3.09 & 25.6 & 1.91 \\
90 & 5.55 & 6.57 & 3.06 & 25.6 & 2.76 \\
\hline
\end{tabular}

The details of the parameter extraction procedure, the analytical dependence of $M$ with the angle, as well as the effects of losses can be found in [41].

\subsection{CPWs Loaded with Pairs of Coupled SRRs}

CPW transmission lines loaded with pairs of SRRs can be used as sensors based on frequency splitting. Typically, the coupling between resonators cannot be neglected due to their close proximity. Thus, the modeling of these loaded lines must include not only the coupling between the line and the 
resonant elements, but also the inter-resonator coupling [26]. A typical CPW loaded with a pair of square-shaped SRRs and its lumped element equivalent circuit are depicted in Figure 13 [26]. Line-to-resonator coupling is accounted for through $M_{1}$ and $M_{2}$, and inter-resonator coupling through $M^{\prime}$. The per-unit-cell inductance and capacitance of the line are $L$ and $C$, respectively, and the SRRs are described by the resonant tanks $L_{1}-C_{1}$ and $L_{2}-C_{2}$. The model considers the general case of an asymmetric structure.
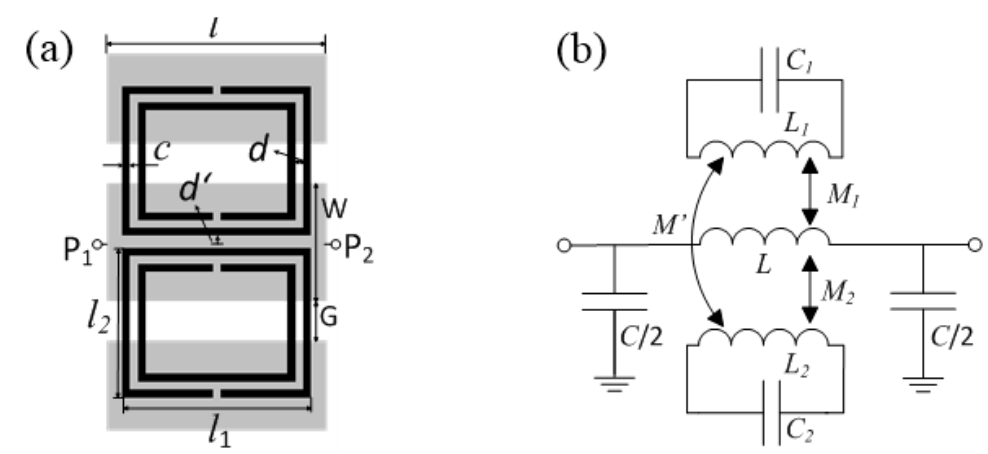

Figure 13. Typical topology of a CPW loaded with a pair of SRRs (a), and lumped element equivalent circuit model, considering magnetic coupling between SRRs (b). The model considers the general case of different SRRs, or identical SRRs with different dielectric loading (in the latter case, $L_{1}=L_{2}$ ).

The circuit of Figure $13 \mathrm{~b}$ is a $\pi$-circuit where the shunt branches are purely capacitive. Thus, the transmission zeros (or resonance frequencies of the coupled resonators) are given by the poles of the series reactance. From Kirchhoff's equations, including the different mutual couplings, applied to the series branch of the circuit of Figure 13b, the impedance of this branch is found to be:

$$
Z_{s}(\omega)=j \omega L+j \omega^{3} \times\left\{\frac{C_{1} M_{1}^{2}\left(1-\frac{\omega^{2}}{\omega_{2}^{2}}\right)+C_{2} M_{2}^{2}\left(1-\frac{\omega^{2}}{\omega_{1}^{2}}\right)+2 \omega^{2} M_{1} M_{2} M^{\prime} C_{1} C_{2}}{\left(1-\frac{\omega^{2}}{\omega_{1}^{2}}\right)\left(1-\frac{\omega^{2}}{\omega_{2}^{2}}\right)-\omega^{4} M^{\prime 2} C_{1} C_{2}}\right\}
$$

where $\omega_{1}=\left(L_{1} C_{1}\right)^{-1 / 2}$ and $\omega_{2}=\left(L_{2} C_{2}\right)^{-1 / 2}$ are the resonance frequencies of the isolated resonators. By forcing the denominator of the last term to be zero, the notch (or transmission zero) frequencies can be derived, namely:

$$
\omega_{ \pm}^{2}=\frac{\omega_{1}^{2}+\omega_{2}^{2} \pm \sqrt{\left(\omega_{1}^{2}-\omega_{2}^{2}\right)^{2}+4 M^{\prime 2} \omega_{1}^{4} \omega_{2}^{4} C_{1} C_{2}}}{2\left[1-M^{\prime 2} \omega_{1}^{2} \omega_{2}^{2} C_{1} C_{2}\right]}
$$

Note that these frequencies do not depend on the mutual couplings, $M_{1}$ and $M_{2}$, between the line and the SRRs, but depend on the mutual coupling, $M^{\prime}$, between the SRRs. If inter-resonator coupling is negligible $\left(M^{\prime}=0\right)$, which is reasonable if the SRRs are separated enough, the solutions of Expression (2) are simply $\omega_{l}$ and $\omega_{2}$. In this case, the equivalent circuit is formally identical to the one reported in [42], but with two different resonators in the series branch, due to the asymmetry of the unit cell with regard to the line axis. 
If $M^{\prime} \neq 0$ and the structure is symmetric $\left(M_{1}=M_{2}=M, \omega_{1}=\omega_{2}=\omega_{0}, L_{1}=L_{2}=L_{r}\right.$, and $\left.C_{1}=C_{2}=C_{r}\right)$, the two solutions of (2) are ( $k_{M}$ is the magnetic coupling coefficient):

$$
\omega_{ \pm}=\frac{\omega_{o}}{\sqrt{1 \mp \frac{M^{\prime}}{L_{r}}}}=\frac{\omega_{o}}{\sqrt{1 \mp k_{M}}}
$$

However, one of the solutions, the one with the $(-)$ sign in the radicand of Expression (3) $\left(\omega_{+}\right)$, is not actually a transmission zero frequency. The reason is that this frequency also nulls the numerator of the last term in Expression (1). By applying l'Hôpital's rule, it follows that the series impedance is finite at the frequency $\omega_{+}$; hence, we can conclude that only one transmission zero, to the left of $\omega_{0}$ (since $M^{\prime}>0$ ), appears in the transmission coefficient for symmetric structures with magnetically coupled SRRs [26].

The transmission zero frequency for the symmetric case, $\omega_{-}$, can alternatively be obtained by applying the magnetic wall concept to the circuit of Figure 13b. Before that, it is necessary to transform the magnetically coupled inductors $L_{r}$ 's of the circuit of Figure $13 \mathrm{~b}$ to the equivalent T-circuit model (see Figure 14) [44]. Notice that the inductance of each resonator at both sides of the symmetry plane (magnetic wall) is given by $L_{r}+M^{\prime}$, from which Expression (3) with the $(+)$ sign results. It should be mentioned that for the symmetric case, the model of [42] provides the same macroscopic results. However, the equivalent inductance of the resonators in the model presented here includes the effects of $M^{\prime}$, i.e., in [42] $M^{\prime}$ is inherently included in the SRR inductance.

(a)

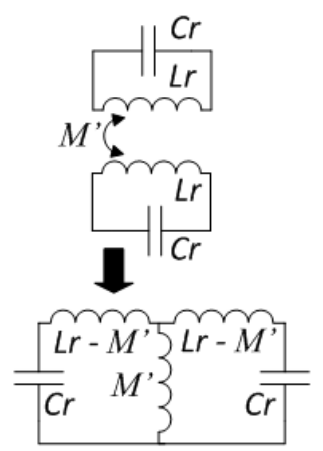

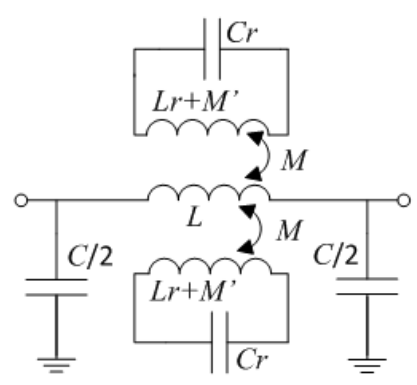

(b)

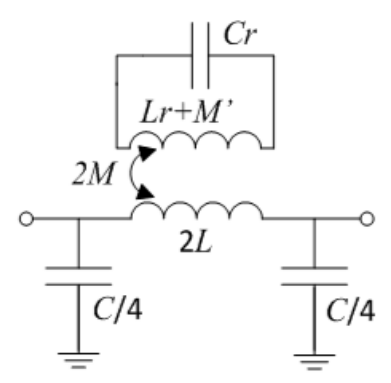

Figure 14. (a) Circuit model of the structure of Figure 13 for the symmetric case, with the inductive coupling between resonators transformed to the equivalent T-model (indicated on the left); (b) circuit that results by applying the magnetic wall concept.

For the asymmetric structure (different coupled SRRs), but considering that their fundamental resonance frequencies are identical $\left(L_{1} \neq L_{2}\right.$ and $C_{1} \neq C_{2}$ where $\left.\omega_{1}=\omega_{2}=\omega_{0}\right)$, Expression (2) gives:

$$
\omega_{ \pm}=\frac{\omega_{o}}{\sqrt{1 \mp \frac{M^{\prime}}{\sqrt{L_{1} L_{2}}}}}=\frac{\omega_{o}}{\sqrt{1 \mp k_{M}}}
$$

Note that Expression (4) is formally identical to Expression (3), but replacing the inductance $L_{r}$ with the geometrical mean of the two inductances $L_{1}$ and $L_{2}$ (both expressions can be written in terms of the magnetic coupling coefficient, $k_{M}$ ). The two frequencies given by Expression (4) null the denominator of the last term in (1), but, in general, none of them nulls the numerator, thus providing two transmission zeros. However, there is a specific condition: 


$$
\frac{M_{1}}{M_{2}}=\sqrt{\frac{L_{1}}{L_{2}}}=\sqrt{\frac{C_{2}}{C_{1}}}
$$

that gives only one transmission zero (at $\omega_{-}$), since the numerator of the last term in (1) is also null at $\omega_{+}$, and the impedance of the series branch is finite at this frequency (as the application of l'Hôpital's rule reveals).

The last analysis corresponds to the asymmetric case with arbitrary resonator frequencies $\left(L_{1} \neq L_{2}\right.$ and $C_{1} \neq C_{2}$ with $\omega_{1} \neq \omega_{2}$ ). In this case, the transmission zeros are given by the two solutions of Expression (2), and the mutual coupling between resonators $\left(M^{\prime}\right)$ enhances (or splits) the distance between the transmission zeros, i.e.,

$$
\omega_{+}^{2}-\omega_{-}^{2}=\frac{\sqrt{\left(\omega_{1}^{2}-\omega_{2}^{2}\right)^{2}+4 M^{\prime 2} \omega_{1}^{4} \omega_{2}^{4} C_{1} C_{2}}}{\left[1-M^{\prime 2} \omega_{1}^{2} \omega_{2}^{2} C_{1} C_{2}\right]}>\omega_{1}^{2}-\omega_{2}^{2}
$$

If the resonance frequencies of the isolated resonators $\left(\omega_{1}\right.$ and $\left.\omega_{2}\right)$ are distant enough $\left(\left|\omega_{1}{ }^{2}-\omega_{2}{ }^{2}\right|>>0\right)$ and $M^{\prime}$ is small (this later condition is very reasonable in the considered structures), the two solutions of Expression (2) can be approximated by:

$$
\omega_{+}=\omega_{1} \sqrt{\frac{1+R \cdot k_{M}^{2}}{1-k_{M}^{2}}}>\omega_{1} \quad \omega_{-}=\omega_{2} \sqrt{\frac{1-(1+R) \cdot k_{M}^{2}}{1-k_{M}^{2}}}<\omega_{2}
$$

where we have considered that $\omega_{1}>\omega_{2}$, and the positive dimensionless factor $R=\left(\omega_{1}{ }^{2} / \omega_{2}^{2}-1\right)^{-1}$ has been introduced to simplify expressions (7). The validity of Expression (7) is subjected to large values of $\omega_{1}^{2}-\omega_{2}^{2}$ (small $R$ ) and small values of $M^{\prime}$ ( or $k_{M}$ ), avoiding negative expressions in the square root of $\omega$ - in Expression (7).

To validate the proposed model, the symmetric case has been first considered, where SRR and CPW dimensions are set to $l_{1}=4.8 \mathrm{~mm}, l_{2}=3.8 \mathrm{~mm}, c=d=0.2 \mathrm{~mm}, l=5.6 \mathrm{~mm}, W=3 \mathrm{~mm}$, and $G=1.01 \mathrm{~mm}$. The lossless electromagnetic simulations (inferred from Agilent Momentum) of the structure $\left(S_{21}\right)$, considering different values of inter-resonator distance, $d$, are depicted in Figure 15 (the considered substrate is Rogers RO3010 with $\varepsilon_{r}=11.2$ and $h=1.27 \mathrm{~mm}$ ). The circuit parameters for the four considered cases (extracted from the method reported in [43]) are shown in Table 2 (analytical models for the SRRs are reported in $[16,45,46]$, but these models are not accurate enough with the presence of the CPW). As $d$ ' increases, the mutual coupling $M$ ' decreases, and the resonance frequency increases. Note that the other circuit parameters do not significantly vary, and the agreement between circuit and electromagnetic simulations in the region of interest is very good, pointing out the validity of the model. The measured responses (shown in Figure 15) are also in good agreement (slight discrepancies are due to losses and fabrication related tolerances).

Finally, we have considered an asymmetric structure with different SRR resonance frequencies. The geometry is as follows: $l=5.6 \mathrm{~mm}, W=3 \mathrm{~mm}, G=1.01 \mathrm{~mm}$; upper SRR: $l_{1}=4.8 \mathrm{~mm}, l_{2}=4.6 \mathrm{~mm}$, $c=d=0.2 \mathrm{~mm}$; lower SRRs: $l_{1}=4.8 \mathrm{~mm}, l_{2}=3.8 \mathrm{~mm}, c=d=0.2 \mathrm{~mm}$. We have obtained the frequency response and the circuit parameters for four different values of $d^{\prime}$. The pair of notches (at $f_{-}=\omega-/ 2 \pi$ and $\left.f_{+}=\omega_{+} / 2 \pi\right)$ are depicted in Figure 16, and verify that their distance increases as $d$ ' decreases, and $f_{-}<f_{2}$ and $f_{+}>f_{1}$, in agreement with Expression (7). 
(a)

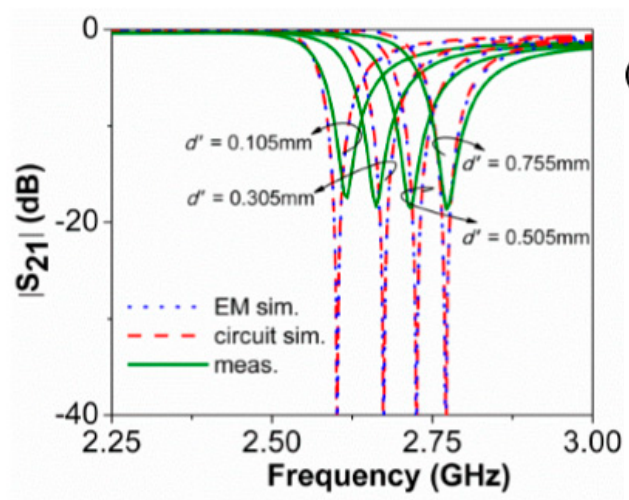

(b)

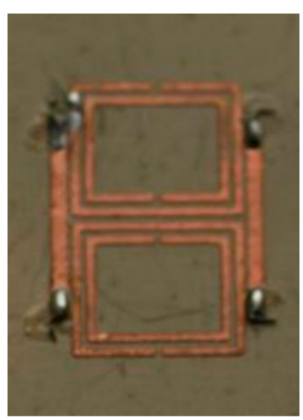

Figure 15. Frequency response (symmetric case) for different values of $d$ ' (a), and detail of one of the fabricated samples (bottom) where the ground planes have been connected through vias and strips to avoid the slot mode (b). From [26]; reprinted with permission from IEEE 2015.

(a)

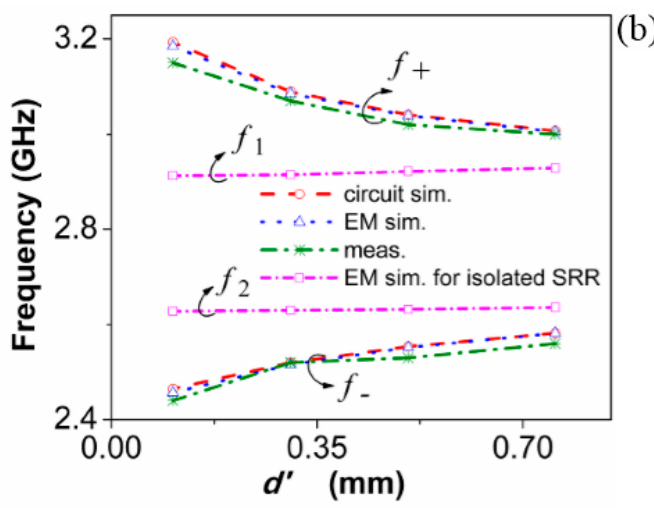

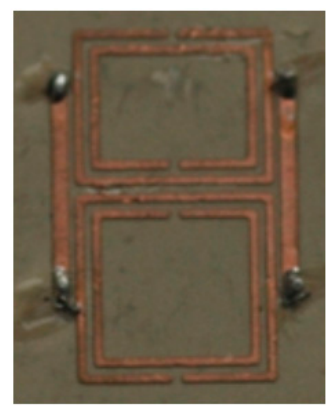

Figure 16. Variation of the notch frequencies as a function of $d$ ' for the asymmetric SRR-loaded CPW (a), and detail of the fabricated sample (bottom face) for $d^{\prime}=0.105 \mathrm{~mm}$ (b). From [26]; reprinted with permission IEEE 2015.

Table 2. Extracted circuit parameters (symmetric case) for different values of $d$ '.

\begin{tabular}{ccccccc}
\hline $\boldsymbol{d}^{\prime}(\mathbf{m m})$ & $\boldsymbol{L} \mathbf{( n H})$ & $\boldsymbol{C}(\mathbf{p F})$ & $\boldsymbol{C}_{\boldsymbol{r}}(\mathbf{p F})$ & $\boldsymbol{L}_{\boldsymbol{r}}(\mathbf{n H})$ & $\boldsymbol{M}(\mathbf{n H})$ & $\boldsymbol{M}^{\prime}(\mathbf{n H})$ \\
\hline 0.105 & 1.82 & 1.58 & 0.44 & 6.85 & 0.82 & 1.74 \\
0.305 & 1.86 & 1.58 & 0.44 & 6.85 & 0.82 & 1.29 \\
0.505 & 1.84 & 1.57 & 0.43 & 6.85 & 0.81 & 1.02 \\
0.755 & 1.85 & 1.55 & 0.43 & 6.85 & 0.80 & 0.80 \\
\hline
\end{tabular}

\section{Applications}

In this section, three applications of transmission lines loaded with resonant elements, where the symmetry related electromagnetic properties are exploited, are presented.

\subsection{Differential Lines and Balanced Filters with Common-Mode Suppression}

It was already pointed out in Section 3.1 that differential lines loaded with CSRRs suppress the common mode whilst the differential mode is kept unaltered. A differential line loaded with CSRRs 
optimized to enhance the common-mode rejection bandwidth is shown in Figure 17 [36]. To widen the rejection bandwidth for the common mode, the CSRRs have been designed with wider rings and inter-ring space $[27,36]$. The target was to achieve at least $20-\mathrm{dB}$ common-mode rejection in the frequency range between $1.2 \mathrm{GHz}$ and $1.8 \mathrm{GHz}$. To this end, three tightly-coupled square-shaped CSRRs suffice to achieve the target specifications. Figure 18 shows the simulated and measured differential- and common-mode insertion loss of the structure. The dimensions of the active region of the structure are $32.8 \mathrm{~mm} \times 10.8 \mathrm{~mm}$, that is $0.43 \lambda \times 0.14 \lambda$. It is remarkable that the measured insertion loss for the differential signal is smaller than $0.5 \mathrm{~dB}$ in the considered range.

(a)

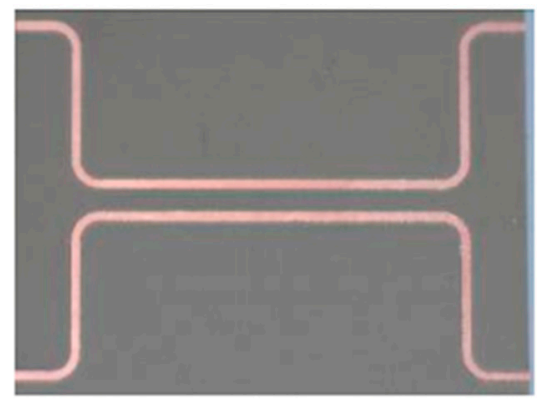

(b)

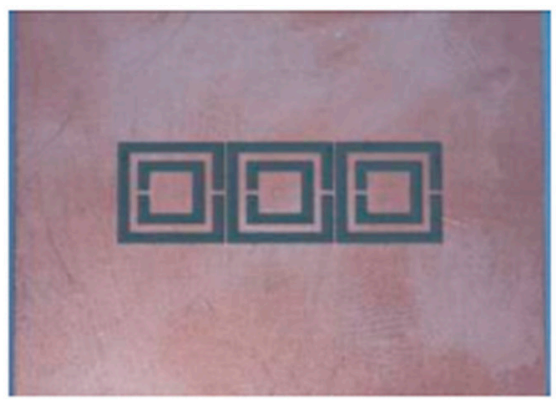

Figure 17. Photograph of the (a) top view and (b) bottom view of the differential line with wideband common-mode rejection. Dimensions are: $c=1.2 \mathrm{~mm}, d=0.8 \mathrm{~mm}, W=1 \mathrm{~mm}$, $S=2.5 \mathrm{~mm}$, and CSRR side length of $10.8 \mathrm{~mm}$. The substrate is Rogers RO3010 with $h=1.27 \mathrm{~mm}, \varepsilon_{r}=10.2$, and loss tangent $\tan \delta=0.0023$.

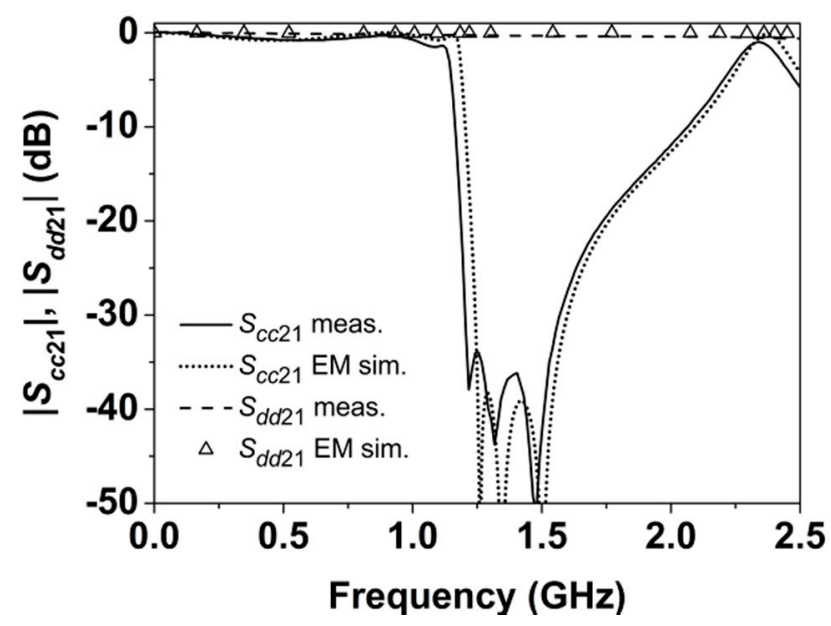

Figure 18. Common-mode and differential-mode insertion loss $\left|S_{c c 21}\right|$ and $\left|S_{d d 21}\right|$ for the structure of Figure 17. From [36]; reprinted with permission from IEEE 2011.

Other common-mode suppressed differential microstrip lines implemented by loading the line with double-slit CSRRs (DS-CSRRs) are reported in [27]. The DS-CSRR is useful to broaden the rejection bandwidth for the common mode, since this particle exhibits a lower inductance than the CSRR, as required for that purpose. For instance, DS-CSRRs have been demonstrated to be useful for the efficient suppression of the common mode in balanced filters [27]. By merely cascading a pair of differential lines, each one loaded with a single DS-CSRR, the suppression of the common mode in differential filters with intrinsic common-mode suppression can be significantly enhanced. This is 
illustrated in Figure 19, where a balanced filter consisting of a pair of coupled stepped impedance resonators (SIRs) fed by a differential line, is depicted. The filter by itself rejects the common mode due to the symmetry of the structure, since the symmetry plane of the resonator exhibits an electric wall at the first SIR resonance. Therefore, such resonators cannot be excited by means of common-mode signals, and the even mode is reflected back to the source due to the presence of the slots between the pair of SIRs. However, the rejection level of the common mode in the region of interest is very limited since it depends on the distance between resonators, and such inter-resonator distance is dictated by filter specifications. However, by cascading DS-CSRRs, the common-mode suppression is severely enhanced, as Figure 20 demonstrates [27].

(a)

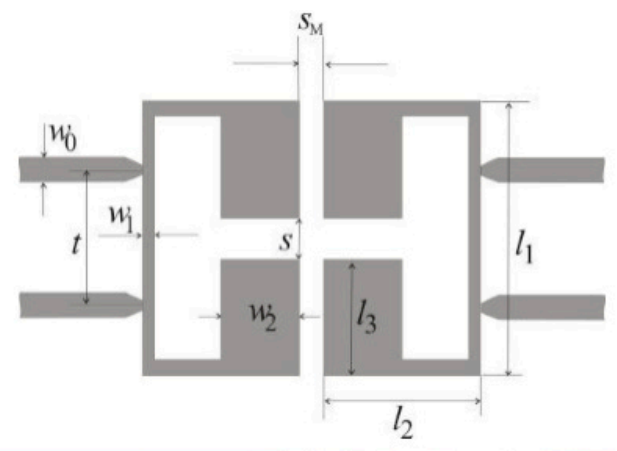

(b)

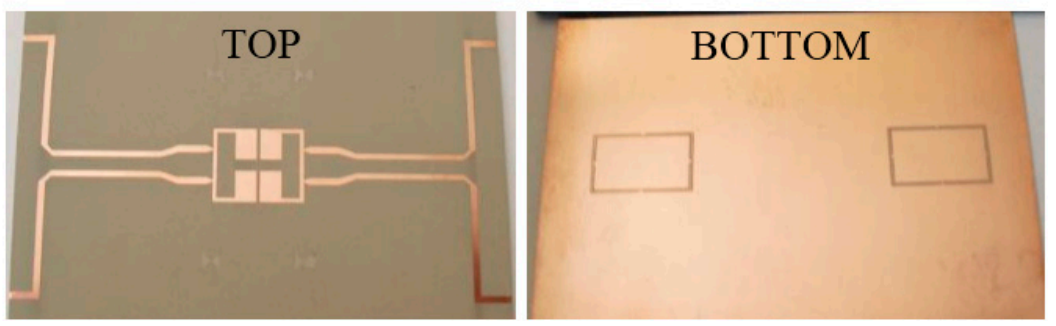

Figure 19. Layout (a) and photograph (b) of the designed differential bandpass filter with improved common-mode rejection. Dimensions are: for the DS-CSRRs, $c=d=0.2 \mathrm{~mm}$, longitudinal side length $=17.6 \mathrm{~mm}$, and transverse side length $=10.8 \mathrm{~mm}$; for the differential line, $W=1 \mathrm{~mm}$, and $S=2.5 \mathrm{~mm}$; for the differential filter, $S_{M}=0.5 \mathrm{~mm}, s=2 \mathrm{~mm}, w_{0}=1.2 \mathrm{~mm}$, $w_{1}=0.7 \mathrm{~mm}, w_{2}=3.7 \mathrm{~mm}, l_{1}=12.8 \mathrm{~mm}, l_{2}=7.5 \mathrm{~mm}, l_{3}=5.4 \mathrm{~mm}$, and $t=5.5 \mathrm{~mm}$. The substrate is Rogers $R O 3010$ with $h=1.27 \mathrm{~mm}, \varepsilon_{r}=10.2$, and $\tan \delta=0.0023$.

The proposed differential filter is a second-order Chebyshev bandpass filter with a central frequency of $1.37 \mathrm{GHz}$, a fractional bandwidth of $10 \%$, and $0.1-\mathrm{dB}$ ripple. The substrate is Rogers RO3010 with $h=1.27 \mathrm{~mm}$, and $\varepsilon_{r}=10.2$. With these specifications and substrate, the layout of the filter is that depicted in Figure 19a (the design of the filter was done following the procedure described in [44]). The frequency response of the filter (differential mode, $S_{d d 21}$ and $S_{d d 11}$ ) is shown in Figure 20. The common-mode insertion loss $\left(S_{c c 21}\right)$, also depicted in the figure, exhibits a rejection level of about $20 \mathrm{~dB}$ in the pass band region. However, by cascading two identical DS-CSRRs as shown in Figure 19b, the common-mode rejection is roughly increased up to $50 \mathrm{~dB}$ in the region of interest (see Figure 20). These results point out that DS-CSRRs provide an efficient path to enhance the common-mode noise rejection in balanced filters. 


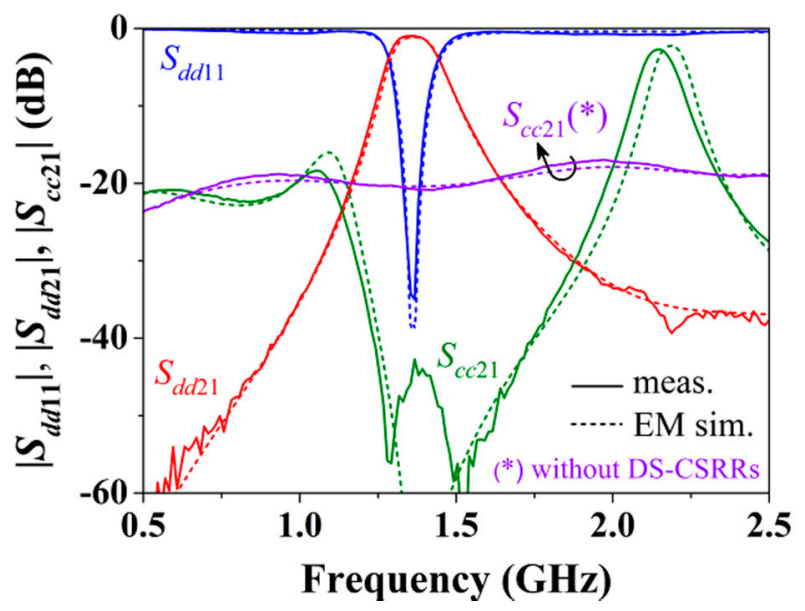

Figure 20. Simulated and measured differential-mode return loss $\left|S_{d d 11}\right|$ and insertion loss $\left|S_{d d 21}\right|$, and common-mode insertion loss $\left|S_{c c 21}\right|$ for the structure of Figure $19 \mathrm{~b}$ with and without the presence of the DS-CSRRs. From [27]; reprinted with permission from IEEE 2012.

\subsection{Angular Displacement and Velocity Sensors}

Resonance-based angular displacement and velocity sensors have been reported in [40,41] and [47] in CPW and microstrip technology, respectively. In all the cases, a circular-shaped ELC resonator and line are used since by this means, linearity is optimized. For the CPW-based sensor, the line is non-uniform along the propagation direction. However, matching is preserved provided the central strip and slot widths have the appropriate dimensions. With regard to the microstrip-based sensor, the transmission line (that behaves like a transducer element) is actually composed of a circular-shaped divider/combiner, as depicted in Figure 21. Let us consider that a circular ELC resonator (attached to the rotating object) is symmetrically placed above a symmetric pair of microstrip lines propagating a common-mode signal. Under these conditions, the symmetry plane (i.e., the midplane) behaves as a magnetic wall. As proven in [47], and also according to the discussion of Subsection 2.1, the structure is transparent to signal transmission if the ELC electric wall is aligned with the symmetry plane of the structure. Contrarily, a transmission zero arises if the alignment is with the ELC magnetic wall. In the former situation, the fundamental ELC resonance cannot be excited. By contrast, the resonator is strongly coupled (magnetically) to the lines if the magnetic walls are aligned. Such behavior, explained through the equivalent circuit models of Subsection 3.2, suggests that rotations with a $90^{\circ}$ dynamic range can be measured from the common-mode transmission coefficient.

By feeding the structure with a single-ended signal, the divider acts as a single-ended to common-mode signal transition. Conversely, the combiner converts the common-mode signal into a single-ended signal. The circular-shaped lines exhibit $50-\Omega$ even-mode characteristic impedance. The combiner/divider is implemented with 35.35- $\Omega$ impedance inverters (quarter-wavelength lines at $1.45 \mathrm{GHz}$ ) to achieve matching to $50-\Omega$ reference ports. The circular $50-\Omega$ line pair (which is the active part of the transducer-like element and hence responsible for resonator coupling) must be arranged face-to-face with the ELC external ring in order to enhance electromagnetic coupling. Furthermore, the line pair as well as the resonator was circular-shaped to enhance linearity, as mentioned above. 
(a)

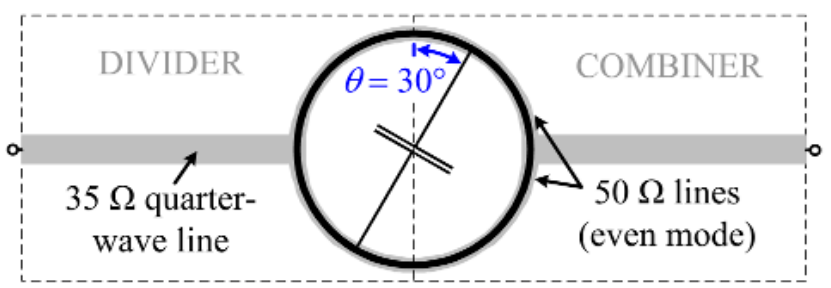

(b)
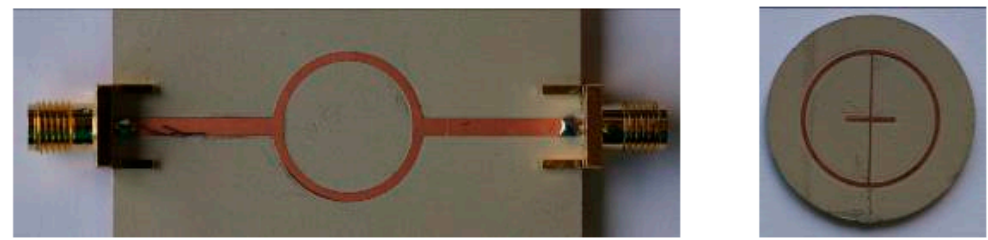

Figure 21. Angular displacement sensor. (a) Layout; and (b) photograph. The substrates are Rogers RO3010 with $\varepsilon_{r}=11.2, h=1.27 \mathrm{~mm}$ (microstrip line) and $h=0.635 \mathrm{~mm}$ (resonator), and $\tan \delta=0.0023$. The line widths $W$ are $2.06 \mathrm{~mm}$ and $1.04 \mathrm{~mm}$. In reference to Figure 9, ELC mean radius $r_{0}=8.05 \mathrm{~mm}, w_{1}=6 \mathrm{~mm}, w_{2}=l_{1}=s=0.2 \mathrm{~mm}$, and $w_{3}=0.5 \mathrm{~mm}$.

The displacement measurement was performed using an experimental set-up similar to that reported in [41]. The ELC substrate was attached to a step motor through a Teflon slab with 3.5-mm thickness and $\varepsilon_{r}=2.08$. The air gap between the ELC and the microstrip lines was set to $0.254 \mathrm{~mm}$. As shown in Figure 22, the rotation angle can be sensed from the notch magnitude (and frequency as well), the dependence being reasonably linear.

(a)

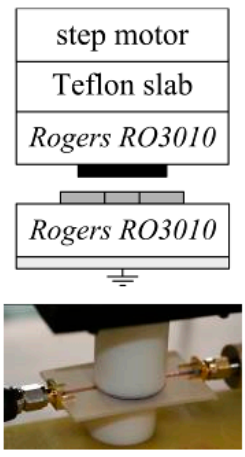

(b)

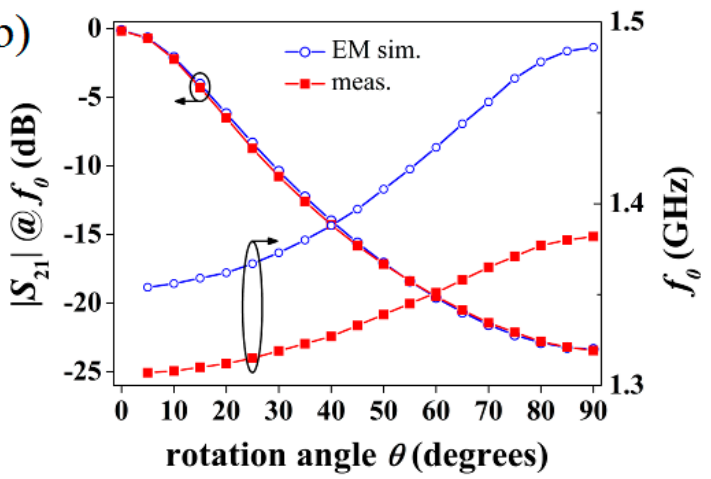

Figure 22. (a) Experimental set-up, and (b) notch magnitude and frequency versus the angular displacement. The frequency shift between measured and simulated data is attributed to substrate- and fabrication-related tolerances $\left(f_{0}\right.$ is very sensitive to the resonator parameters). From [47]; reprinted with permission IEEE 2014.

Let us now consider that the ELC is continuously rotating. To obtain the angular velocity, $\omega_{r}$, a harmonic signal tuned at a fixed frequency (e.g., the notch frequency corresponding to $\theta=10^{\circ}$, $f_{0}=1.308 \mathrm{GHz}$ ) is injected to the input port, whereas an isolator plus an envelope detector are cascaded to the output port. For two different angular velocities, the envelope signals at the output (which are dependent on the transmission coefficient) are captured by an oscilloscope (Figure 23), and the velocities are derived from the time difference between consecutive transmission peaks, $T_{m}=T_{r} / 2=1 / 2 f_{r}$ $\left(\omega_{r}=2 \pi f_{r}\right)$. By configuring the step motor with $f_{r}=1 \mathrm{~Hz}$ and $50 \mathrm{~Hz}$ (i.e. nominal values), the measured 
velocities are $f_{r}=0.998 \mathrm{~Hz}$ and $50.251 \mathrm{~Hz}$, respectively, validating the approach. Rotation speeds as high as required can be measured by means of this approach since the carrier signal frequency (within the microwave region of the electromagnetic spectrum) is much higher than the angular velocity of actual rotating systems. Precision can be improved by merely averaging the time between peaks. Other related sensors for measuring angular spatial variables are reported in [48-50].

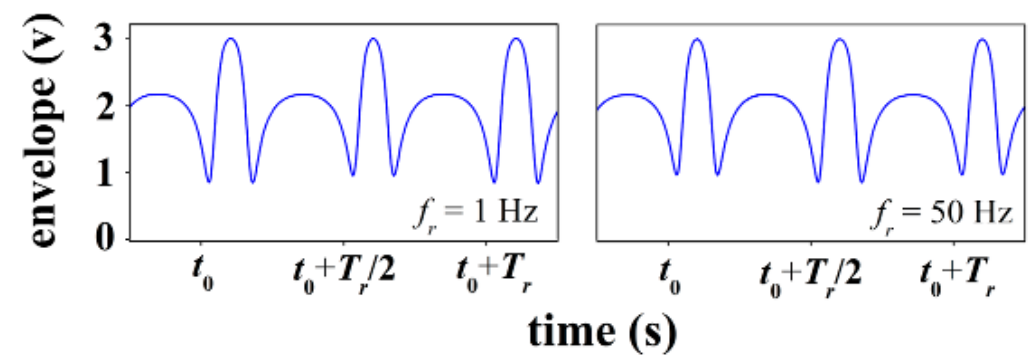

Figure 23. Angular velocity measurements for an arbitrary initial time to. From [47]; reprinted with permission from IEEE 2014.

\subsection{Differential Permittivity Sensors and Comparators}

The last reported application of this paper concerns frequency splitting sensors for dielectric measurements. The idea in these sensors, which are based on a transmission line loaded with a pair of resonant elements in a symmetric configuration, is to sense by inspection of the resonance frequencies (number and shift). If symmetry is preserved, a single notch in the transmission coefficient appears, as discussed in Section 3.3 in reference to CPWs loaded with SRRs. However, if symmetry is disrupted, frequency splitting occurs, with the result of two notches. Since symmetry can be perturbed by several means (by linear or by angular displacements, by asymmetric dielectric loading, etc.), different physical variables can be measured. Thus, this type of frequency-splitting structures can be used as displacement sensors [35], or as sensors/comparators for dielectric characterization, among others. In the latter case, the one of interest in this section, a pair of dielectric samples is loaded onto the structure (interestingly, one of them can be a reference and well-known sample). Using this approach, a differential permittivity sensor may be designed. Alternatively, a comparison (differential by definition) between the two samples may be readily performed; if these loads are identical, a single notch appears, whereas two separated resonances are expected if they are different (note that in a comparator only the number of resonance frequencies is of interest).

These dielectric comparators can be implemented by means of SRR-loaded CPW transmission lines. However, the proof-of-concept comparator illustrated in this section, firstly reported in [34], is based on a pair of shunt-connected stepped-impedance resonators (SIRs) loaded to a CPW or microstrip transmission line. Like in CPWs loaded with pairs of SRRs, there is magnetic coupling between the SIRs loaded in pairs [34]. Indeed, the expression providing the resonance frequencies is identical to that reported in Section 3.3 (Equation (2)). Nevertheless, there are some differences between the two topologies; one is relative to the position of the resonance frequency in the presence of inter-resonator coupling (above or below the intrinsic resonance frequency of the resonant element) for the symmetric configuration; another is in regard to the fact that under certain conditions a single notch is possible for asymmetric structures in SRR-loaded lines, but not in SIR-loaded lines. These details are treated in depth 
in $[26,34,51]$. The interest here is thus to present a proof-of-concept comparator for dielectric measurements. It was implemented by loading a conductor-backed CPW (CB-CPW) with two identical SIRs. The structure was measured through on-wafer microwave probes without any dielectric load, and with a piece of glass $(\varepsilon r=4.6)$ on top of one of the SIRs (see Figure 24). The appearance of a lower transmission zero in the latter case caused by the symmetry perturbation validates experimentally the sensing principle indicating that different dielectric loads are present on top of the two SIRs.

(a)
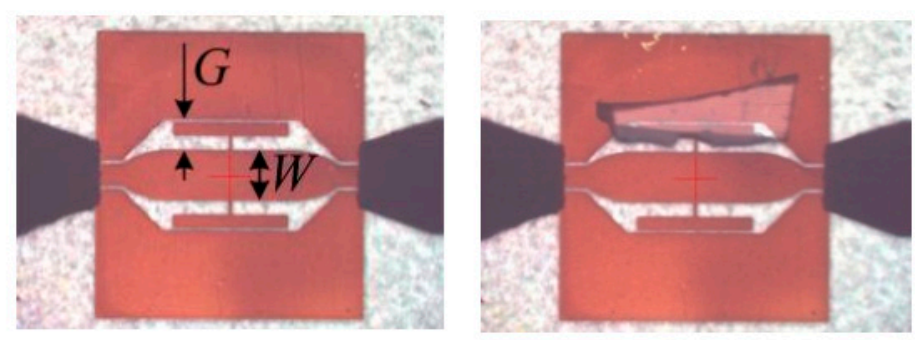

(b)

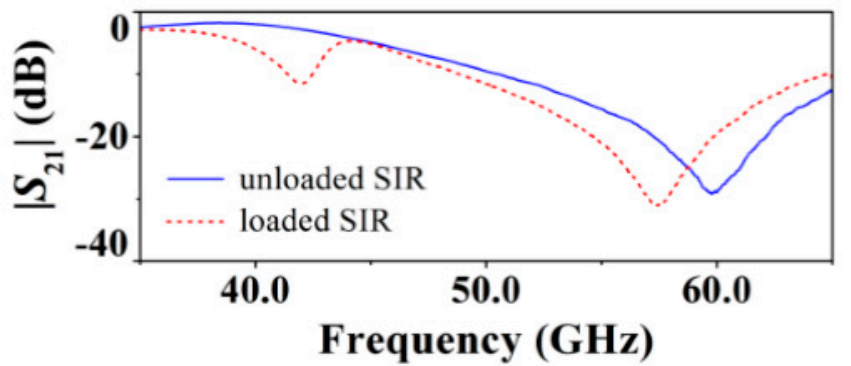

Figure 24. (a) Photograph of the proof-of-concept demonstrator without (left) and with (right) a piece of glass on the top; (b) measured transmission coefficient. CPW dimensions are: $W=350 \mu \mathrm{m}$ and $G=210 \mu \mathrm{m}$. SIR dimensions are: $l_{1}=90 \mu \mathrm{m}$ and $l_{2}=100 \mu \mathrm{m}$, and $w_{1}=800 \mu \mathrm{m}, w_{2}=20 \mu \mathrm{m}$ ( $l$ and $w$ are the length and width of the sections, respectively). The substrate parameters are $\varepsilon_{r}=4.6, h=200 \mu \mathrm{m}$, and $\tan \delta=0.0021$. From [34]; reprinted with permission from IEEE 2014.

It is worth mentioning that dielectric microwave sensors for analysis of biological cells and organic tissue or for the determination of component concentration/composition in aqueous (or other liquid) solutions by means of microfluidics, mostly based on frequency shift (i.e., not based on symmetry properties), have been proposed recently [18,21,52-62].

\section{Conclusions}

In conclusion, it has been shown in this paper that symmetry can be exploited for the design of microwave components, such as common-mode suppressed differential lines, and different types of sensors. Specifically, the considered devices are based on transmission lines loaded with planar resonant elements. Two types of structures have been utilized: resonance-based structures and frequency-splitting structures. In resonance-based structures, the lines are loaded with a single resonant element, and the line is transparent if the structure is fully symmetric and the symmetry plane of the line and resonator are of distinct electromagnetic structure (namely, one an electric wall and the other one a magnetic wall). Accordingly, the coupling between the line and the resonator is cancelled, and the resonator is not 
excited. However, when symmetry is perturbed, the resonator is effectively coupled to the line and a notch in the transmission coefficient appears. On the other hand, in frequency-splitting structures, where the line is loaded with two identical resonators, the resonance frequency is split into two frequencies when symmetry is disrupted. Otherwise, a single notch in the transmission coefficient appears. The circuit models of several illustrative structures of these types of resonator-loaded lines have been presented and discussed. Finally, some representative applications have been reported, including differential lines and filters with common-mode suppression, and sensors for measuring angular displacements and velocities as well as for differential permittivity measurements.

\section{Acknowledgments}

This work has been supported by Spain-MICIIN (contracts TEC2010-17512, TEC2013-40600-R, and CSD2008-00066) and by FEDER funds. Thanks are given to the Catalan Government for giving support through the project 2014SGR-157. Ferran Martin is in debt to Institució Catalana de Recerca i Estudis Avançats (ICREA) for being awarded with an ICREA Academia distinction.

\section{Author Contributions}

Jordi Naqui and Ferran Martin conceived the principle pointed out in this work for the control of the transmission characteristics in transmission lines loaded with symmetric configurations of resonant elements. Jordi Naqui and Lijuan Su made the simulations and experimental characterization of the fabricated prototypes, and validated the circuit models as well, jointly with Javier Mata. Jordi Naqui and Ferran Martín wrote the paper.

\section{Conflicts of Interest}

The authors declare no conflict of interest.

\section{References}

1. Makimoto, M.; Yamashita, S. Compact bandpass filters using stepped impedance resonators. Proc. IEEE 1979, 67, 16-19.

2. Pendry, J.B.; Holden, A.J.; Robbins, D.J.; Stewart, W.J. Magnetism from conductors and enhanced nonlinear phenomena. IEEE Trans. Microwave Theory Tech. 1999, 47, 2075-2084.

3. Sauviac, B.; Simovski, C.R.; Tretyakov, S.A. Double split-ring resonators: analytical modeling and numerical simulations. Electromagnetics 2004, 24, 317-338.

4. Somovski, C.R.; Sauviac, B. Role of wave interaction of wires and split-ring resonators for the losses in a left-handed composite. Phys. Rev. E 2004, 70, 46607.

5. Safwat, A.M.E.; Tretyakov, S.A.; Räisänen, A. Dual bandstop resonator using combined split ring resonator and defected ground structure. Microw. Opt. Tech. Lett. 2007, 49, 1249-1253.

6. Baena, J.D.; Marqués, R.; Medina, F.; Martel, J. Artificial magnetic metamaterial design by using spiral resonators. Phys. Rev. B 2004, 69, 014402. 
7. Falcone, F.; Lopetegi, T.; Baena, J.D.; Marqués, R.; Martín, F.; Sorolla, M. Effective negative- $\varepsilon$ stop-band microstrip lines based on complementary split ring resonators. IEEE Microwave Wireless Compon. Lett. 2004, 14, 280-282.

8. Ahn, D.; Park, J.-S.; Kim, C.-S.; Kim, J.; Qian, Y.; Itoh, T. A design of the low-pass filter using the novel microstrip defected ground structure. IEEE Trans. Microwave Theory Tech. 2001, 49, 86-93.

9. Lim, J.-S.; Kim, C.-S.; Lee, Y.-T.; Ahn, D.; Nam, S. A spiral-shaped defected ground structure for coplanar waveguide. IEEE Microwave Wireless Compon. Lett. 2002, 12, 330-332.

10. Safwat, A.M.E.; Podevin, F.; Ferrari, P.; Vilcot, A. Tunable bandstop defected ground structure resonator using reconfigurable dumbbell-shaped coplanar waveguide. IEEE Trans. Microwave Theory Tech. 2006, 54, 3559-3564.

11. Safwat, A.M.E.; Tretyakov, S.; Räisänen, A.V. Defected ground and patch-loaded planar transmission lines. IET Microw. Antennas Propag. 2009, 3, 195-204.

12. Bonache, J.; Martín, F.; Gil, I.; García-García, J.; Marqués, R.; Sorolla, M. Microstrip bandpass filters with wide bandwidth and compact dimensions. Microwave Opt. Technol. Lett. 2005, 46, 343-346.

13. Wu, B.; Liang, C.-H.; Li, Q.; Qin, P.-Y. Novel dual-band filter incorporating defected SIR and microstrip SIR. IEEE Microwave Wireless Compon. Lett. 2008, 18, 392-394.

14. García-García, J.; Martín, F.; Falcone, F.; Bonache, J.; Baena, J.D.; Gil, I.; Amat, E.; Lopetegi, T.; Laso, M.A.G.; Marcotegui-Iturmendi, J.A.; et al. Microwave filters with improved stop band based on sub-wavelength resonators. IEEE Trans. Microwave Theory Tech. 2005, 53, 1997-2006.

15. Gil, M.; Bonache, J.; Martín, F. Metamaterial filters: A review. Metamaterials 2008, 2, 186-197.

16. Marqués, R.; Martín, F.; Sorolla, M. Metamaterials with Negative Parameters: Theory, Design and Microwave Applications; John Wiley: New York, NY, USA, 2008.

17. Mandel, C.; Kubina, B.; Schüßler, M.; Jakoby, R. Passive chipless wireless sensor for two-dimensional displacement measurement. In Proceedings of the Microwave Conference (EuMC), 2011 41st European, Manchester, UK, 10-13 October 2011; pp. 79-82.

18. Puentes, M.; Weiss, C.; Schüßler, M.; Jakoby, R. Sensor array based on split ring resonators for analysis of organic tissues. In Proceedings of the Microwave Symposium Digest (MTT), 2011 IEEE MTT-S International, Baltimore, MD, USA, 5-10 June 2011.

19. Naqui, J.; Durán-Sindreu, M.; Martín, F. Novel sensors based on the symmetry properties of split ring resonators (SRRs). Sensors 2011, 11, 7545-7553.

20. Chen, T.; Li, S.; Sun, H. Metamaterials application in sensing. Sensors 2012, 12, 2742-2765.

21. Withayachumnankul, W.; Jaruwongrungsee, K.; Tuantranont, A.; Fumeaux, C.; Abbott, D. Metamaterial-based microfluidic sensor for dielectric characterization. Sens. Actuat. A Phys. 2013, 189, 233-237.

22. Preradovic, S.; Balbin, I.; Karmakar, N.C.; Swiegers, G.F. Multiresonator-based chipless RFID system for low-cost item tracking. IEEE Trans. Microwave Theory Tech. 2009, 57, 1411-1419.

23. Preradovic, S.; Chandra-Karmakar, N. Chipless RFID: Bar code of the future. IEEE Microwave Mag. 2010, 11, 87-98.

24. Naqui, J.; Durán-Sindreu, M.; Martín, F. Modeling split ring resonator (SRR) and complementary split ring resonator (CSRR) loaded transmission lines exhibiting cross polarization effects. IEEE Antennas Wirel. Propag. Lett. 2013, 12, 178-181. 
25. Marqués, R.; Medina, F.; Rafii-El-Idrissi, R. Role of bi-anisotropy in negative permeability and left handed metamaterials. Phys. Rev. B 2002, 65, 144441.

26. Su, L.; Naqui, J.; Mata-Contreras, J.; Martín, F. Modeling metamaterial transmission lines loaded with pairs of coupled split ring resonators. IEEE Anten. Wirel. Propag. Lett. 2015, 14, 68-71.

27. Naqui, J.; Fernández-Prieto, A.; Durán-Sindreu, M.; Mesa, F.; Martel, J.; Medina, F.; Martín, F. Common mode suppression in microstrip differential lines by means of complementary split ring resonators: Theory and applications. IEEE Trans. Microwave Theory Tech. 2012, 60, 3023-3034.

28. Naqui, J.; Durán-Sindreu, M.; Martín, F. Selective mode suppression in coplanar waveguides using metamaterial resonators. Appl. Phys. A-Mater. Sci. Process 2012, 109, 1053-1058.

29. Naqui, J.; Durán-Sindreu, M.; Martín, F. Selective mode suppression in microstrip differential lines by means of electric-LC (ELC) and magnetic-LC (MLC) resonators. Appl. Phys. A: Mater. Sci. Process. 2014, 115, 637-643.

30. Naqui, J.; Durán-Sindreu, M.; Martín, F. Alignment and position sensors based on split ring resonators. Sensors 2012, 12, 11790-11797.

31. Horestani, A.; Fumeaux, C.; Al-Sarawi, S.; Abbott, D. Displacement sensor based on diamond-shaped tapered split ring resonator. IEEE Sens. J. 2013, 13, 1153-1160.

32. Horestani, A.K.; Naqui, J.; Abbott, D.; Fumeaux, C.; Martín, F. Two-dimensional displacement and alignment sensor based on reflection coefficients of open microstrip lines loaded with split ring resonators. Electron. Lett. 2014, 50, 620-622.

33. Naqui, J.; Martín, F. Mechanically reconfigurable microstrip lines loaded with stepped impedance resonators and potential applications. Int. J. Antennas Propag. 2014, doi:10.1155/2014/346838.

34. Naqui, J.; Damm, C.; Wiens, A.; Jakoby, R.; Su, L.; Martín, F. Transmission lines loaded with pairs of magnetically coupled stepped impedance resonators (SIRs): Modeling and application to microwave sensors. In Proceedings of the Microwave Symposium (IMS), 2014 IEEE MTT-S International, Tampa, FL, USA, 1-6 June 2014.

35. Horestani, A.K.; Naqui, J.; Shaterian, Z.; Abbott, D.; Fumeaux, C.; Martín, F. Two-dimensional alignment and displacement sensor based on movable broadside-coupled split ring resonators. Sens. Actuat. A Phys. 2014, 210, 18-24.

36. Naqui, J.; Fernández-Prieto, A.; Durán-Sindreu, M.; Selga, J.; Medina, F.; Mesa, F.; Martín, F. Split rings-based differential transmission lines with common-mode suppression. In Proceedings of the Microwave Symposium Digest (MTT), 2011 IEEE MTT-S International, Baltimore, MD, USA, 5-10 June 2011.

37. Baena, J.D.; Bonache, J.; Martín, F.; Marqués, R.; Falcone, F.; Lopetegi, T.; Laso, M.A.G.; García, J.; Gil, I.; Flores-Portillo, M.; et al. Equivalent circuit models for split ring resonators and complementary split rings resonators coupled to planar transmission lines. IEEE Trans. Microwave Theory Tech. 2005, 53, 1451-1461.

38. Bonache, J.; Gil, M.; Gil, I.; Garcia-García, J.; Martín, F. On the electrical characteristics of complementary metamaterial resonators. IEEE Microwave Wireless Compon. Lett. 2006, 16, 543-545.

39. Schurig, D.; Mock, J.J.; Smith, D.R. Electric-field-coupled resonators for negative permittivity metamaterials. Appl. Phys. Lett. 2006, 88, 041109. 
40. Naqui, J.; Durán-Sindreu, M.; Martín, F. Transmission lines loaded with bisymmetric resonators and applications. In Proceedings of the Microwave Symposium Digest (IMS), 2013 IEEE MTT-S International, Seattle, WA, USA, 2-7 June 2013.

41. Naqui, J.; Martín, F. Transmission lines loaded with bisymmetric resonators and their application to angular displacement and velocity sensors. IEEE Trans. Microwave Theory Tech. 2013, 61, 4700-4713.

42. Aznar, F.; Bonache, J.; Martín, F. Improved circuit model for left handed lines loaded with split ring resonators. Appl. Phys. Lett. 2008, 92, 043512.

43. Aznar, F.; Gil, M.; Bonache, J.; Baena, J.D.; Jelinek, L.; Marqués, R.; Martín, F. Characterization of miniaturized metamaterial resonators coupled to planar transmission lines. J. Appl. Phys. 2008, 104, 114501.

44. Hong, J.-S.; Lancaster, M.J. Microstrip Filters for RF/Microwave Applications; John Wiley: New York, NY, USA, 2001.

45. Bilotti, F.; Toscano, A.; Vegni, L.; Aydin, K.; Alici, K.; Ozbay, E. Equivalent-circuit models for the design of metamaterials based on artificial magnetic inclusions. IEEE Trans. Microwave Theory Tech. 2007, 55, 2865-2873.

46. Ramaccia, D.; di Palma, L.; Ates, D.; Ozbay, E.; Toscano, A.; Bilotti, F. Analytical model of connected bi-omega: Robust particle for the selective power transmission through sub-wavelength apertures. IEEE Trans. Ant. Prop. 2014, 62, 2093-2101.

47. Naqui, J.; Martín, F. Angular displacement and velocity sensors based on electric-LC (ELC) loaded microstrip lines. IEEE Sens. J. 2014, 14, 939-940.

48. Horestani, A.; Abbott, D.; Fumeaux, C. Rotation sensor based on horn-shaped split ring resonator. IEEE Sens. J. 2013, 13, 3014-3015.

49. Ebrahimi, A.; Withayachumnankul, W.; Al-Sarawi, S.; Abbott, D. Metamaterial-inspired rotation sensor with wide dynamic range. IEEE Sens. J. 2014, 14, 2609-2614.

50. Sipal, V.; Narbudowicz, A.; Ammann, M.J. Contactless measurement of angular velocity using circularly polarized antennas. IEEE Sens. J. 2014, doi:10.1109/JSEN.2014.2385815.

51. Naqui, J.; Su, L.; Mata, J.; Martín, F. Analysis of transmission lines loaded with pairs of coupled resonant elements and application to sensors. J. Magn. Magn. Mater. 2015, 383, 144-151.

52. Boybay, M.S.; Ramahi, O.M. Material characterization using complementary split-ring resonators. IEEE Trans. Instrum. Measur. 2012, 61, 3039-3046.

53. Albishi, A.M.; Ramahi, O.M.; Boybay, M.S. Complementary split-ring resonator as a high sensitivity sensor. In Proceedings of the Antennas and Propagation Society International Symposium (APSURSI), 2012 IEEE, Chicago, IL, USA, 8-14 July 2012.

54. Ebrahimi, A.; Withayachumnankul, W.; Al-Sarawi, S.; Abbott, D. High-sensitivity metamaterial-inspired sensor for microfluidic dielectric characterization. IEEE Sens. J. 2014, 14, 1345-1351.

55. Abduljabar, A.; Rowe, D.; Porch, A.; Barrow, D. Novel microwave microfluidic sensor using a microstrip split-ring resonator. IEEE Trans. Microwave Theory Tech. 2014, 62, 679-688.

56. Chretiennot, T.; Dubuc, D.; Grenier, K. A microwave and microfluidic planar resonator for efficient and accurate complex permittivity characterization of aqueous solutions. IEEE Trans. Microwave Theory Tech. 2013, 61, 972-978. 
57. Grenier, K.; Dubuc, D.; Chen, T.; Artis, F.; Chretiennot, T.; Poupot, M.; Fournie, J. Recent advances in microwave-based dielectric spectroscopy at the cellular level for cancer investigations. IEEE Trans. Microwave Theory Tech. 2013, 61, 2023-2030.

58. Chen, T.; Artis, F.; Dubuc, D.; Fournie, J.J.; Poupot, M.; Grenier, K. Microwave biosensor dedicated to the dielectric spectroscopy of a single alive biological cell in its culture medium. In Proceedings of the Microwave Symposium Digest (IMS), 2013 IEEE MTT-S International, Seattle, WA, USA, 2-7 June 2013.

59. Chretiennot, T.; Dubuc, D.; Grenier, K. Optimized electromagnetic interaction microwave resonator/microfluidic channel for enhanced liquid bio-sensor. In Proceedings of the Microwave Conference (EuMC), 2013 European, Nuremberg, Germany, 6-10 October 2013; pp. 464-467.

60. Artis, F.; Dubuc, D.; Fournie, J.J.; Poupot, M.; Grenier, K. Microwave dielectric bio-sensing for precise and repetitive living cells suspension analysis. In Proceedings of the Microwave Conference (EuMC), 2013 European, Nuremberg, Germany, 6-10 October 2013; pp. 468-470.

61. Chretiennot, T.; Dubuc, D.; Grenier, K. Double stub resonant biosensor for glucose concentrations quantification of multiple aqueous solutions. In Proceedings of the Microwave Symposium (IMS), 2014 IEEE MTT-S International, Tampa, FL, USA, 1-6 June 2014.

62. Puentes, M. Planar Metamaterial Based Microwave Sensor Arrays for Biomedical Analysis and Treatment; Springer: New York, NY, USA, 2014.

(C) 2015 by the authors; licensee MDPI, Basel, Switzerland. This article is an open access article distributed under the terms and conditions of the Creative Commons Attribution license (http://creativecommons.org/licenses/by/4.0/). 